\title{
Das Mysterienwesen im Kontext VON Religion Und Gesellschaft deR RÖMISCHEN KAISERZEIT
}

\author{
Daniel Mielke \\ Technische Universität Darmstadt / Universität Potsdam
}

Recibido: 01-11-2013 | Evaluado: 28-11-2013 | Aprobado: 28-11-2013

ZuSAMMENFASSUNG: Das Mysterienwesen war ein integraler Bestandteil des reichhaltigen Angebots an Sinngebungsmodellen im Römischen Kaiserreich. Es bestach durch ganz eigene Qualitäten (besonders: dieswie jenseitiger göttlicher Beistand) und differierte (angeblich) recht umfassend von einem jahrhundertealten Verstehens-, Vorstellungssowie Erwartungshorizont.

Mein Promotionsvorhaben, dessen Aufbau hier konzis dargestellt wird, möchte $\mathrm{zu}$ einer historisch ausgewogeneren Betrachtung der

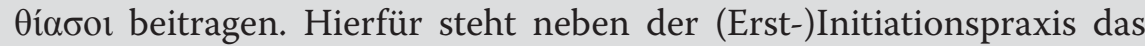
innergemeindliche Miteinander im Zentrum der typologisierenden Untersuchung. Primär ausgehend von den "Metamorphoses" des Apuleius von Madaura, wird gezeigt, dass althergebrachte Erwartungen sowie Ansichten auch elementar für die collegia waren. Ferner gilt besonderes Augenmerk grundsätzlichen Problemen beim Umgang mit dem Mysterienwesen sowie der Religionsgeschichtlichen Schule.

Schlüsselworte: Kaiserzeitliches Mysterienwesen - (Erst-)Initiationspraxis - sacra publica - soziales Distinktionspotenzial - „Vergöttlichung“ des Mysten.

ABSTRACT: In the Roman Empire the mystery cults were an integral part within the extensive range of options giving meaning, orientation and guidance. The communities stood out due to specific qualities and are said to differ comprehensively from several centuries-old conventions and attitudes.

$\mathrm{My} \mathrm{PhD}$ project, whose structure is shown concisely, wants to contribute to a more balanced historical consideration of the fíaбot. Besides the 
(first) initiation practice the typological investigation centres on the circles' togetherness. Primarily emanating from the "Metamorphoses" of Apuleius of Madaura, it is shown that certain traditional expectations as well as opinions were elementary for the collegia, too. Furthermore, special attention is drawn to the basic problems dealing with the ancient mystery cults and the Religionsgeschichtliche Schule.

Keywords: Mystery cults in the Roman Empire - (first) initiation practice sacra publica - potential of social distinction - „deification“ of the mystes.

\section{Das Thema}

$\mathrm{D}$ e antike Welt war eine Welt voller Götter. Nicht nur die Kapitolinische Trias, Priapus, die Lares, Herkules, Sol Invictus, JHWH oder der in den Augen der meisten Reichsbewohner zunächst noch recht junge Gott der Christen gehörten schließlich zum großen religiösen Spektrum der Kaiserzeit. Viele Zeitgenossen wussten auch etwas mit mehr oder weniger obskuren Göttern, wie z. B. Isis, Mithras oder Neos Glykon, anzufangen. Spätestens seit dem Hellenismus gehörte das bis in die Spätantike hinein wirkmächtige Mysterienwesen zum reichhaltigen Angebot an Sinngebungsmodellen, die schließlich auch überall im Römischen Weltreich Halt sowie Orientierung versprachen. ${ }^{1}$ Rom hatte im Zuge seiner imperialen Machtausdehnung verschiedene Unsterbliche kennen gelernt ${ }^{2}$ und diese, wie etwa die aus Phrygien

* Der vorliegende Beitrag, Anfang Oktober 2013 verfasst, basiert auf einem Vortrag, den ich im Sommersemester 2013 im Altertumswissenschaftlichen Kolloquium der Klassischen Philologie und der Alten Geschichte der Universität Potsdam halten konnte. Für kritische Hinweise und weiterführende Denkanstöße danke ich besonders Pedro A. Barceló, Manfred Clauss und Michael Stahl sowie Eike Faber.

1. Dass die collegia zum farbenprächtigen Markt mehr oder minder suspekter Ideen gehörten, die dem Menschen z. B. inneren Frieden, übermenschlichen Beistand im Dies- und Jenseits o. ä. in Aussicht stellten, betont u. a. Walter Burkert, Antike Mysterien: Funktionen und Gehalt, C. H. Beck'sche Verlagsbuchhandlung (Oscar Beck), München, 1990, S.11: „In den früheren Epochen scheinen verschiedene Formen der »Religion«, einschließlich der Verehrung neuer und fremder Götter und auch die Einrichtung besonderer »Mysterien «, nebeneinander zu stehen, ohne sich auszuschließen; sie erscheinen als Spielformen, Optionen, ja Moden innerhalb des einen uneinheitlichen und doch kontinuierlichen Konglomerats, das wir die Religion der Antike nennen." [Hervorhebungen im Original, D. M.]. Auch bspw. Iuv. VI 511-594. illustriert das facettenreiche Allerlei an stadtrömischen Sinngebungsmodellen.

2. Den hier skizzierten Zusammenhang stellt besonders gut Pedro A. BARCEló, Kleine römische Geschichte, Wissenschaftliche Buchgesellschaft, Darmstadt, 2005, S.88. dar: „Der Einzug des Apollonkultes, das Heimischwerden der kleinasiatischen Mater Magna oder die Verbreitung der orientalischen Mysterienreligionen, um nur einige Beispiele zu erwähnen, markierten stets bestimmte Phasen der römischen Großmachtbildung. Im ständigen Kreislauf des Gebens und Nehmens gab es eine sichtbare und eine verborgene Linie: Rom exportierte Soldaten, Provinzialadministratoren, Händler sowie bildungshungrige Aristokraten und importierte im Gegenzug Sklaven und Reichtümer, griechische Philosophie und Lebensart, hellenistische Technik und Wissenschaft, nicht zuletzt auch Kulte aus allen Gegenden seines Wirkungsraumes." 
stammende Kybele, bisweilen sogar unter seine Staatsgötter aufgenommen (191 v. Chr.). ${ }^{3}$ Voraussetzung hierfür war, dass sie ihre Göttlichkeit durch verschiedene Machtdemonstrationen hatten beweisen können. ${ }^{4}$

Wodurch zeichneten sich aber die Mysteriengemeinden aus? ${ }^{5}$ Die autonomen Bruderschaften wussten geradezu mit einem ganzen Katalog von konkreten Aussichten und Erwartungen zu begeistern. ${ }^{6}$ Dieser befriedigte

3. Z. B. Liv., XXIX 10, 4. - 11, 8. und 14, 1-14. zeigt, wie Kybele zu Zeiten des Zweiten Punischen Krieges ins römische Bewusstsein trat und was den Beginn ihrer späteren Aufnahme ins Pantheon markiert. Dass sich die Römische Republik nicht nur Kybeles Beistands versichern wollte, sondern noch andere Motive der evocatio zugrunde lagen, stellt u. a. DARJA ŠTERBENC ERKER, Religiöse Rollen römischer Frauen in "griechischen“ Ritualen, Franz Steiner Verlag, Stuttgart, 2013, S.247. heraus: „Die Einführung war eine Integration der »phrygischen« Kybele aus Kleinasien in die öffentliche Religion Roms, als die »res publica« ihre Expansionsabsichten und hegemonistischen Ambitionen auf dieses Gebiet formulierte." [Hervorhebungen im Original, D. M.]

4. Dass man göttliches Wirken allem voran an der Natur bzw. an natürlichen Prozessen, die dem damaligen Verstehen weithin verschlossen blieben, glaubte ausmachen zu können, verdeutlicht Cic. nat. deor. I 39. betont.

5. In der Forschung wird nur noch selten zur Beschreibung des Mysterienwesens auf den Begriff „(orientalische) Mysterienreligionen“ benutzt. Warum man letzten Endes von „-religion“ Abstand nehmen sollte, lassen die Ausführungen von z. B. Marion Giebel, Das Geheimnis der Mysterien. Antike Kulte in Griechenland, Rom und Ägypten, Patmos Verlag GmbH \& Co. KG, München, 2003, S.13f. erkennen: „Es gab keine zentrale Hierarchie, keine Glaubensartikel, somit auch keine Ketzer. Insofern hat man zu Recht gesagt, daß es sich hier um keine Religionen in dem festumgrenzten Sinne handelt wie beim Judentum, Christentum und Islam. Der allgemein übliche Wortgebrauch »Mysterienreligionen « - bereits in der Antike sprachen Heiden wie Christen von »religio « für einzelne Kulte - sollte also mit den entsprechenden Modifizierungen verstanden werden." [Hervorhebungen im Original, D. M.]. Etwa mit WERNER DAHLHEIMS: Die Welt zur Zeit Jesu, Verlag C. H. BeckohG, München, 2013 liegt eine Veröffentlichung neueren Datums vor, die vereinzelt von „Mysterienreligion(en)“ (S.331/ 334.) respektive "-kulte“ (S.332/ 396.) spricht. Bezug nehmend auf zentrale Überlegungen von Franz-Valéry-Marie Cumont arbeitet u. a. CHRISTIAN WitSCHEL: „Orientalische Kulte" im römischen Reich - neue Perspektiven der altertumswissenschaftlichen Forschung. - In: Blömer, Michael u. a. (Hrsgg.): Iuppiter Dolichenus. Vom Lokalkult zur Reichsreligion, Mohr Siebeck, Tübingen, 2012, S.13-38. heraus, weshalb die Kategorie „orientalisch“ o. ä. zur Beschreibung der Glaubensoptionen vermieden werden sollte ( $v g l$. S.13-19).

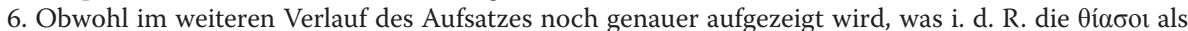
solche ausmachte, möchte ich schon an dieser Stelle auf Überlegungen CARSTEN COLPES, Mysterienkult und Liturgie. Zum Vergleich heidnischer Rituale und christlicher Sakramente. - In: ders. u. a. (Hrsgg.): Spätantike und Christentum: Beiträge zur Religions- und Geistesgeschichte der griechisch-römischen Kultur und Zivilisation der Kaiserzeit, Akad. Verl., Berlin, 1992, S.203-228, hier: S.222. hinweisen: „Mysterienreligionen sind [...] solche, die Geheimnisse enthalten und enthalten wollen, konkret: die ihre Rituale und Lehren, die meist mythologische Form haben, geheim halten. Bestimmte Gemeinschaften taten dies ersichtlich deshalb, weil sie einen besonderen Heilsbesitz damit verbanden. Es folgte daraus, daß sie besondere Bedingungen setzen mußten, deren Erfüllung Voraussetzung dafür war, daß sie auch Außenstehenden Anteil an diesem Heilsbesitz gaben, und daß sie diese Anteilsgabe in besondere Einweihungsprozeduren kleideten, welche den Heilsweg symbolisieren, den ein Mensch von außen in ihr Inneres zurücklegt. Und da die Gemeinschaft selbst Ort des Heiles war, das sie jedoch symbolisch transzendiert, führt der Heilsweg nicht nur in die Gemeinde hinein, sondern zur Erlösung überhaupt. Arkandisziplin, Initiationspraxis und Wiedergeburtsvorstellungen gelten deshalb weithin als die drei Merkmale, an denen man eine Mysterienreligion erkennt." Wie jedoch bspw. neben Plut. mor. 578b. besonders JAN N. Bremmer, Religious Secrets and Secrecy in Classical Greece. - In: Kippenberg, Hans G. u. a. (Hrsgg.): Secrecy and Concealment. Studies in the History of Mediterranean and Near Eastern Religions, E. J. Brill, Leiden u. a., 1995, S.61-78. zeigt, kann man die von COLPE zusammengestellten Indizien nicht rundheraus als Alleinstellungsmerkmale antiker Mysteriengemeinden verstehen. Besonders deutlich weist CARL SCHNEIDER, Kulturgeschichte des Hellenismus, Bd. 2, C. H. Beck'sche Verlagsbuchhandlung (Oscar Beck), München, 1969, S.869. auf

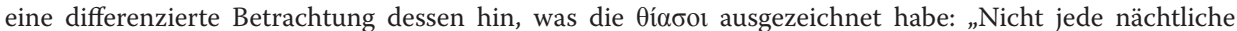
Geheimfeier bedeutet schon einen Hinweis auf eine Mysterienreligion, ebensowenig jede lokale geheime religiöse Überlieferung oder jeder lokale Heroenkult, selbst dann nicht, wenn er einige Züge aus der 
verschiedene persönliche Vorstellungen. Hierzu gehörte gerade der einem jeden Mitglied gewisse göttliche Beistand, der sich auf das Dies- wie Jenseits erstreckte. ${ }^{7}$ Ferner boten sie eine, gemessen an den staatstragenden sacra publica, vermeintlich ungleich innigere persönliche Beziehung mit dem Unsterblichen $^{8}$ oder heimatliche Geborgenheit in einer relativ elitären Gruppe Gleichgesinnter. ${ }^{9}$ Auch konnten die collegiati an zuweilen opulenten Mahlzeiten teilnehmen, die sich oft von der Ärmlichkeit des eigenen Alltags abhoben, ${ }^{10}$ oder man zog persönliche Vorteile aus der Zugehörigkeit zu einem bestimmten collegium ziehen. ${ }^{11}$

Mysterienfrömmigkeit und ihren kultischen Ausdrucksformen angenommen hat." Dass die Arkandisziplin zum antiken Mysterienwesen gehörte, versteht sich von selbst, begegnet uns u. a. in Hom. h. Dem. 478f. oder Apul. met. XI 23. und wird in allen Publikationen zum Thema unterschiedlich erörtert. Um verschiedene ihrer Aspekte besser zu erfassen, bietet sich etwa eine Beobachtung von GIEBEL, Geheimnis (wie Anm. 5), S.15. an: „Das »heilige Schweigen« betraf freilich nur die tiefsten Geheimnisse, zu deren Verständnis eine besondere Unterweisung und Einführung nötig war, nicht aber den Mythos des Gottes und seine Kultfeiern im allgemeinen. [...]. Der magische Charakter, der Ritualen mit heiligen Namen und Gebräuchen stets anhaftet, verbietet deren Profanierung. Zum anderen diente eine solche Geheimhaltung dazu, die Solidarität der Gruppe nach innen zu verstärken und die Autorität und Attraktivität des Kultes nach außen zu erhöhen." [Hervorhebung im Original, D. M.]

7. Beispielhaft hierfür Apul. met. XI 5f. Da es sich bei den collegia um autonome Bruderschaften handelte, kann nicht verallgemeinert werden, was das jeweilige collegium seinen Mitgliedern verhieß. Hierauf weist z. B. SCHNEIDER, Kulturgeschichte (wie Anm. 6), S.767. hin: „Erst im Hellenismus rückte die »Erlösungsfrömmigkeit« in das Zentrum des religiösen Lebens. [...]. Erlösung kann, je nach Niveau des einzelnen, Befreiung von Angst, Leid, Elend und Tod, sie kann aber auch Befreiung von Unwissenheit und Dummheit, von innerer Unruhe infolge der Doppelnatur des Menschen, Befreiung der Seele vom oder im Leib, Überwindung der niederen Natur des Menschen, und schließlich Befreiung des Göttlichen im Menschen aus einer irgendwie gearteten Gefangenschaft sein. Dazu trat der ganze Komplex der Sehnsucht nach Erlösung von menschlicher Schuld, was auch immer der einzelne oder eine Gemeinschaft darunter verstehen mochte."

8. Z. B. mit Blick auf Plaut. Rud. 694-701. spreche ich mich jetzt schon für eine umsichtigere Bewertung der römischen Staatsgottheiten aus, konnten doch auch sie beim Menschen persönliche Gefühle wie etwa Dankbarkeit oder Hoffnung wecken. U. a. JAime Alvar, Romanising oriental Gods. Myth, Salvation, and Ethics in the Cults of Cybele, Isis, and Mithras, Koninklijke Brill NV, Leiden, 2008, S.212. wendet sich gegen anderslautende und z. T. immer noch vorgebrachte Annahmen, : „It used to be taken as an article of faith that official or civic religion in antiquity was largely distinct from the true feelings of the people." Besondere Beachtung verdienen in diesem Zusammenhang Ausführungen von STEFAN KRAUTER, Bürgerrecht und Kultteilnahme. Politische und kultische Rechte und Pflichten in griechischen Poleis, Rom und antikem Judentum, Walter de Gruyter GmbH \& Co. KG, Berlin u. a., 2004, S.24, die die (über) individuelle Dimension dies- wie jenseitig orientierter Hoffnungen thematisieren. Eindrucksvoll präsentiert er diese als seit jeher zum Menschen gehörend. Anderslautende Annahmen führen laut ihm „letztlich zu der unsinnigen Vorstellung, antike und moderne Menschen seien nicht nur kulturell, sondern in ihrer psychischen Grundausstattung verschieden; antike Menschen seien zu individuellen religiösen Erfahrungen, wie sie moderne Menschen haben könnten, nicht fähig gewesen."

9. Beispielhaft hierfür Apul. met. XI 26. Auf das hier Geschilderte zielt auch eine Überlegung von Maarten J. Vermaseren, Mithras. Geschichte eines Kultes, W. Kohlhammer GmbH, Stuttgart, 1965, S.43. ab. So erklärt er für die Syndexioi, die in einem in London 1954 gefundenen Mithräum ab ca. 150 n. Chr. zusammenkamen: „Die Darstellung des thrakischen Reitergottes, der genau wie Mithras als Sonnengott verehrt wurde, legt die Vermutung nahe, daß etliche Londoner Gemeindemitglieder aus den Balkanländern stammten." Dass bestimmte Ethnien in einer anderen Umgebung am eigenen Brauchtum festhielten, zeigt ferner bspw. Strab. XVII 1, 12.

10. Beispielhaft hierfür Apul. met. XI 24.

11. Dass man den persönlichen Aufstieg entweder auf das direktes göttliches Eingreifen zurückführte bzw. Vorteile aus der Mitgliedschaft zu einer bestimmten sodalitas ziehen konnte, geht u. a. aus Apul. met. XI 28/30. hervor. So erklärt etwa Ulrike EgelhaAf-Gaiser, Kulträume im römischen Alltag. Das Isisbuch des Apuleius und der Ort von Religion im kaiserzeitlichen Rom, Franz Steiner Verlag, Stuttgart, 2000, 


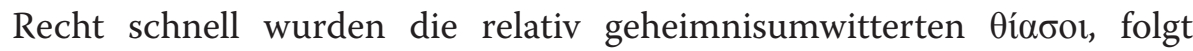
man etwa Franz-Valéry-Marie Cumont, zu den "gefährlichsten und hartnäckigsten Gegner[n] des Christentums [...]. ${ }^{{ }^{112}}$ Als solche wurden sie zum Gegenstand gezielter christlicher Anfeindungen. In erster Linie lag dies daran, dass sie ihren Mitgliedern sowohl ein besseres nachtodliches Dort als auch die Emanzipation von den unentrinnbaren Schicksalsmächten zusicherten. ${ }^{13}$ Es bestand eine gerade von den christlichen Apologeten als unsäglich empfundene, ja geradezu als diabolisch angesehene Nähe besonders zu den eigenen Verheißungen und Kultvollzügen. ${ }^{14}$

Bei den staatlich sanktionierten sodalitates ${ }^{15}$ handelte es sich um verhältnismäßig exklusive Gemeinschaften. Zugang hierzu erlangte man mittels mehr oder minder anspruchsvoller Initiationsprozeduren. Viele kannten ein mehrstufiges Weihesystem: ${ }^{16}$ Der zum véo $\alpha^{\alpha} v \eta ́ \rho$ erhobene Adorant ${ }^{17}$ erhielt

S.103/106: „Praktisch sind die religiöse und berufliche Karriere, Kultpraxis und Alltagsleben eng verflochten und bedingen einander gegenseitig. [...]. Obwohl die Mysterienweihen einen religiösen Statuswechsel, ja im Ausnahmefall sogar eine Karriere im Isiskult einleiten und damit einen konkreten Eigenwert in der Kultorganisation und Hierarchiebildung haben, dienen sie doch primär der Selbstbestätigung und psychischen Stärkung des Kultanhängers für eine erfolgreiche Bewältigung des Alltags. Die von Lucius gewählte religiöse Lebensform des Mysterienanhängers ist bei genauer Betrachtung nicht diametral verschieden zum profanen Lebensalltag, sondern dessen komplementärer Bestandteil: Die religiöse und berufliche Karriere, der Isispriester und Staatsanwalt bilden eine untrennbare Einheit. Und trotz des propagierten Lebensideals der Abkehr von den materiellen, weltlichen Gütern bleiben der eigene Ruhm und das gesellschaftliche Ansehen Maßstab für eine »objektive« Bewertung der getroffenen Lebenswahl des Kultanhängers." [Hervorhebung im Original, D. M.]

12. Franz-VAlÉRY-Marie Cumont: Die orientalischen Religionen im römischen Heidentum, Wissenschaftliche Buchgesellschaft, Darmstadt, 1989, S.13.

13. Dass sich der Mensch als ohnmächtig dem willkürlich wie rätselhaft verstandenen Schicksal ausgeliefert empfand, geht u. a. aus Plin. nat. II 7. hervor. Aus der großen Menge zeitgenössischer Hoffnungen auf den umfassenden göttlichen Beistand sei an dieser Stelle Apul. met. XI 15. genannt.

14. Iust. Mart. apol. I 66f. stellt ein beredtes Zeugnis dafür dar, dass die Christen zentrale Inhalte sowie Riten bestimmter Mysterienlehren (hier: Mithraismus) kategorisch zurückwiesen und sie als diabolisch o. ä. verunglimpften. Worauf sich die christliche Polemik im Wesentlichen konzentrierte, kann bspw. RichARD L. Gordon, s. v. Mithras. - In: RAC 24 (2012), Sp.964-1009, hier: Sp.1000. entnommen werden und wird etwa anhand von Firm. err. V 2. deutlich: „Insofern Mysterienkulte in apologetischem Zusammenhang vorkommen, werden sie wegen der Immoralität der Götter, Blutvergießen, u. weltlicher Vergnügungen angegriffen."

15. Beispielhaft hierfür ILS 4175. Anhand kurzer Beispiele für das staatliche Vorgehen gegen bestimmte Sinngebungsmodelle fasst HeNDRIK S. VERSNEL, Römische Religion und religiöser Umbruch. - In: Vermaseren, Maarten J. (Hrsg.): Die orientalischen Religionen im Römerreich, E. J. Brill, Leiden, 1981, S.41-66, hier: S.50f. die staatliche Handlungsmaxime wie folgt zusammen: „Sie zeigen den Staat in Realisierung der traditionellen Ideologie, die vorschrieb, das Alte zu bewahren und das Neue nicht abzuweisen - vorausgesetzt, das Neue ließ sich in bestehende Strukturen und Lebensanschauung einfügen -, allen desintegrierenden und entwurzelnden religiösen Neuerungen aber mit Argwohn und Repression zu begegnen." U. a. aus Cic. nat. deor. III 5f. geht hervor, dass das sakrosankte Traditum auch im religiösen Bereich als Richtschnur allen pflichtbewussten wie zweckorientierten Handelns verstanden wurde und bspw. Hor. carm. III 6, 1-8. belegt, dass zwischen dem rechten Umgang mit den übermenschlichen Gewalten und dem Wohlergehen des Weltreiches ein Kausalzusammenhang bestand.

16. Z. B. die in Synes. Dion X 6. genutzten Anlehnungen an die Eleusinischen Mysterien unterstreichen, dass man hier zwei Weihestufen kannte.

17. Folgende Beobachtung von Émile Durкheim, Die Grundformen des religiösen Lebens. - In: Fürstenberg, Friedrich (Hrsg.): Religionssoziologie, Luchterhand, Neuwied (Rhein) u. a., 1970, S.35-55, hier: S.35f. bietet sich an, um sich eine prinzipielle Vorstellung davon zu machen, was den gottbehüteten véos ỏví $\rho$ als solchen ausgezeichnet haben mag: „Der Gläubige, der mit seinem Gott kommuniziert hat, ist nicht nur ein Mensch, der neue Wahrheiten sieht, die der Ungläubige nicht kennt, er ist auch ein Mensch, der 
dank der Gottheit bzw. mithilfe seines Mystagogen ${ }^{18}$ sukzessive Einblicke in ein exklusives Weltverstehen. Ihm wurde ein Wissen anthroposophischtheosophisch-kosmologischer Natur zuteil. ${ }^{19}$ Überdies war es dem (werdenden) sodalis möglich, sowohl gemeindeintern als auch coram publico mit den ihm eigenen Möglichkeiten zu imponieren und so zu Ansehen zu gelangen. Immerhin hatte er vor seiner Aufnahme und im Rahmen seiner Mitgliedschaft recht beträchtliche Opfer auf sich zu nehmen. Exempli gratia wurde (während der Vorbereitungen auf die Weihen) ein bestimmter Lebensstil verlangt ${ }^{20}$ oder das Gemeindeleben bzw. die öp $\gamma 1 \alpha$ zeichneten sich durch mehr oder weniger kostspielige Unternehmungen (z. B. Bankette etc. ${ }^{21}$ aus. Demnach bewirkte jede

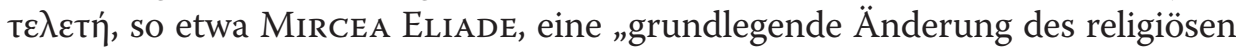
und gesellschaftlichen Status des Einzuweihenden [...]."22 Somit privilegierte die Zugehörigkeit zu einem Oíaбos den Neophyten nicht nur mit einem quasi rechtlich sanktionierten Anspruch auf transzendenten Beistand ${ }^{23}$ und forderte ihn fortan verschiedentlich, ${ }^{24}$ sondern amortisierte sich in recht unmittelbarer Form, nämlich in Gestalt praktischer Lebenshilfe, Geselligkeit, Teilnahme an Festmählern, Prestigegewinn usw.

mehr »vermag«. Er verspürt in sich mehr Kraft, sei es, um die Schwierigkeiten des Daseins zu ertragen, sei es, um sie zu überwinden. Er ist gleichsam emporgehoben über das menschliche Elend, weil er über sein Mensch-Sein emporgehoben wird; er glaubt sich vom Übel errettet, unter welcher Form er übrigens auch immer das Übel begreifen mag." [Hervorhebung im Original, D. M.]

18. Bspw. Apul. met. XI 27f. legt den Schluss nahe, dass die Mystagogen verschiedene pastorale Tätigkeiten ausübten. Neben den göttlichen Heilszusagen unterstreicht dies, dass der einzelne gerade in den $\theta i ́ \alpha \sigma o \imath$ zum Gegenstand persönlicher Fürsorge wurde. Richard Reitzenstein, Die hellenistischen Mysterienreligionen. Nach ihren Grundgedanken und Wirkungen, Wissenschaftliche Buchgesellschaft, Darmstadt, 1966, S.20. etwa zeigt anhand von Apul. met. XI 25, dass sich eine enge persönliche Beziehung zwischen ihnen und den Adepten entwickeln konnte: „Priesterliche Abkunft wird für ihn [sc. Lucius] durch die Fiktion dargestellt, daß der Weihende, indem er den Unterricht erteilt, zum Vater des Initianden wird."

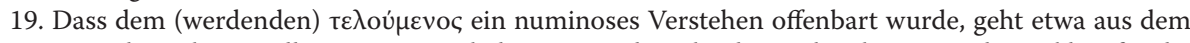
figurativen Gebrauch spezieller Mysterieninhalte u. a. in Plat. Phaidr. 250 b c. hervor und so erklärt für die Eleusinischen Mysterien z. B. Karl KerÉnyi, Die Mysterien von Eleusis, Rhein-Verlag AG, Zürich, S.30: „Ein beglückendes Wissen besaß der Eingeweihte nicht erst da drüben, sondern beides, Glückseligkeit und Wissen, waren seit der Schau schon hier sein Eigentum.“

20. Sehr eindrucksvoll geht aus der Zusammenschau von Tert. ieiun. XVI, Tib. I 3, 23-26. und Apul. met. XI 19. hervor, dass bspw. (werdende) Isiasten sexuell abstinent zu leben hatten, um so die für die Weihe(n) obligatorische Katharsis zu erlangen.

21. Beispielhaft hierfür ApUl. met. XI 23f.

22. Mircea Eliade, Das Mysterium der Wiedergeburt. Versuch über einige Initiationstypen, Insel Verlag, Frankfurt (Main), 1988, S.11. U. a. Diod. V 49, 6. zeigt, dass sich der (werdende) Myste in seinem Gestus verändern konnte.

23. Als geradezu klassisches Beispiel für das Dargestellte sei hier HoM. H. DEM. 480-482/486-489. genannt.

24. Beispiele für Verpflichtungen, die die Mitgliedschaft nach sich ziehen konnte und die uns u. a. recht deutlich etwa in CIL IV 787. begegnen, listet etwa BurKerT, Mysterien (wie Anm. 1), S.47f/50. auf: „Man trifft sich zu gemeinsamen kultischen Unternehmungen, insbesondere zu Opfern mit dem zugehörigen Gemeinschaftsmahl, auch zu öffentlichen Prozessionen, "pompai«, die der städtischen Öffentlichkeit demonstrieren, wer zur Gruppe gehört. Gegenseitige Verpflichtungen, wie sie auch zu privater »Freundschaft« gehören, werden übernommen, Unterstützung in Prozessen und vor allem Teilnahme am Begräbnis eines Mitglieds. Von weiteren Unternehmungen sozialer Hilfe verlautet nichts; [...]. In sehr viel weltlicherem Kontext erscheint der Terminus [sc. Isiakos bzw. Isiakoi] in Pompeii, inmitten von Wahlparolen: »die Gesamtheit der Isisanhänger schlägt Cn. Helvius Sabinus als Ädil vor«, [...]. Hier läßt sich eine religiös verbundene Gruppe auf Politik ein oder läßt sich zumindest von einem Politiker gebrauchen;" [Hervorhebungen im Original, D. M.] 
Genau wie die meisten gottesdienstlichen Handlungen gingen die Initiationsriten i. d. R. auf einen iєpóৎ $\lambda o ́ \gamma o \varsigma$ zurück. Durch die anamnetische Begehung ${ }^{25}$ boten sie die Möglichkeit zum Heilserwerb. Besonders gut stellt Hans-Josef KLAUCK das soeben Skizzierte dar: Die Vergegenwärtigung ausgesuchter Passagen des aitiologischen Mythos ermöglichte es, „dass der Einzuweihende hineingenommen wird in das Götterschicksal, Anteil gewinnt an ihren Mühen und vor allem an ihrem Sieg. So kommt eine rituelle Partizipation zustande, die in sich die Aussicht auf Gewinn von Heil, $\sigma \omega$ тпрí $\alpha$, birgt." ${ }^{26}$

Durch den bspw. als Palingenese ${ }^{27}$ verstandenen Schritt unterschied man sich hinfort von sämtlichen Nicht-Initiierten. Diese Abgesondertheit

25. Folgende Überlegung von Karl Prümm, Der christliche Glaube und die altheidnische Welt, Bd. 2, Verlag Jakob Hegner, Leipzig, 1935, S.311, die sich mit Prud. perist. X 1011-1050. in Verbindung bringen

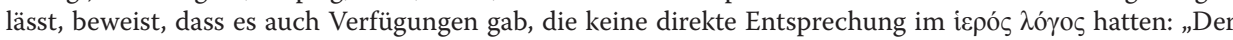
Taurobolienbrauch steht ja zunächst überhaupt »in keinem sichtlichen Zusammenhang mit irgendeinem bestimmten Göttermythos«. In dem Kult der Göttermutter und des Attis ist er gar nicht bodenständig. [...]. Die Anlage des Ritus selbst läßt aber nichts vom Prinzip der Nachfolge des Kultheros erkennen.“ [Hervorhebung im Original, D. M.] U. a. Dieter Zeller, Sterbende Götter als Identifikationsfiguren. In: BiKi 45 (1990), S.132-139. skizziert, dass sodalitates ihre Kulthandlungen sowie den Göttermythos bisweilen (adressatenorientiert) erweiterten. Gerade mit Bezug auf Firm. err. XXII 3. zeigt er, dass christliche Vorstellungen (konkret: Grablegung Jesu nach der Kreuzigung, Gedenken der Grabesruhe Jesu und spätere Feier der Auferstehung) übernommen werden konnten (vgl. S.137.). Dass die jeweilige sodalitas hiermit weder die eigentlichen Interpretationen noch Hoffnungen verband, erörtert insbesondere HaNsJosef KLauck, Die religiöse Umwelt des Urchristentums, Bd. 1, W. Kohlhammer GmbH, Stuttgart u. a., S.127: „Vom Sterben und wieder Aufleben der Mysteriengottheiten redet man heute mit sehr viel mehr Zurückhaltung als zu Zeiten Brückners und Casels, gleichsam in Anführungszeichen. Der Typos ist in sich differenzierter zu sehen, manche Göttergestalten sind davon gar nicht betroffen, und etwas der christlichen Auferstehungshoffnung genau Vergleichbares liegt nirgends vor [...].”

26. Ders., Die antiken Mysterienkulte und das Urchristentum. Anknüpfung und Widerspruch. - In: ZMR 1 (2002), S.3-25, hier: S.12. HoM. H. DEM. 268-274. ist beispielhaft dafür, dass Kulthandlungen den

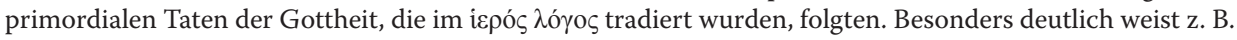
Ov. fast. IV 535f. darauf hin, dass etwaige Vollzüge bewusst das sakrosankte Traditum rezipierten.

27. Mit Apul. met. XI 23f. sei hier auf einen locus classicus dafür verwiesen, wie die Neuschöpfung des Mysten, der sich dies- wie jenseitiger Privilegien sicher sein konnte, bisweilen inszeniert und kommuniziert wurde. Äußerst prägnant fasst Claus-Peter März, „Selig, wer jenes geschaut.... Initiation und Initiationsriten bei den Mysterien. - In: BiKi 45 (1990), S.125-131, hier: S.129. das hier Dargestellte zusammen: „Mit dem Initianden geht somit in der zentralen Initiationshandlung eine Verwandlung vor sich: Er stirbt seinem alten Leben ab und wird als ein nunmehr unter dem Schutz der Isis Stehender neu

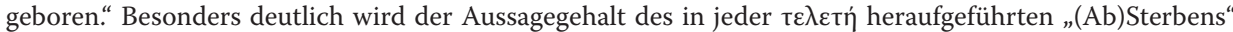
bei Eliade, Wiedergeburt (wie Anm. 22), S.14: „Für das archaische Denken bringt nichts deutlicher die Idee eines »Endes«, der endgültigen Vollendung einer Sache zum Ausdruck als der Tod - [...]." [Hervorhebung im Original, D. M.]. Wie tief die besonders in Apul. met. XI 24. dargestellte Verbindung des Initiierten mit dem Gott nun gewesen sein mag, erörtert u. a. AleXANDER J. M. WedDerburn, Baptism and Resurrection. Studies in Pauline Theology against its Graeco-Roman Background, J. C. B. Mohr (Paul Siebeck), Tübingen, 1987, S.337/340/342: „Such wearing of divine dress, we may suggest, symbolizes not so much deification or admittance to the ranks of gods as fellowship with the gods, dedication and belonging to the one whose garb is worn, and at the same time the possession in one's life of the divine qualities and powers characteristic of that deity. [...]. Rather these badges marked the initiate's or priest's belonging to the god, being under

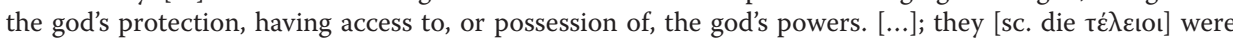
»identified « with that deity, but not in the fullest or most strict sense; rather they were at one with the deity, communing with it and at the same time representing it to their fellow mortals." [Hervorhebung im Original, D. M.] 
tritt besonders deutlich durch die obligatorische Arkandisziplin hervor, ${ }^{28}$ die bestimmte Inhalte betraf (besonders: die welterklärende Interpretation der gemeindeeigenen Kulterzählung sowie die hierauf mehrheitlich zurückgehenden ö $\rho \gamma(\alpha) .{ }^{29}$ Sie begegnet uns weiterhin in der Überzeugung der (werdenden) collegiati, nur sie seien in Form des göttlichen Beistands privilegiert. „Doch wer die Opfer nicht darbringt, oder sie meidet, wird niemals [t]eilhaft solchen Glückes; er vergeht in modrigem Düster. [...]. Hochselig die Erde bewohnenden Menschen [d]enen die beiden sich gütig und liebend erzeigen; sie schicken Plutos bald in ihr großes Haus als Genossen am Herde; [d]er aber stiftet reiches Vermögen den sterblichen Menschen“"30, so etwa der pseudohomerische Demeterhymnus über die exklusiven Heilszusagen an die Teilnehmer der Eleusinien.

Weiterhin mag der Umstand, dass bestimmte gottesdienstliche Handlungen der uneingeweihten Öffentlichkeit entzogen waren, ${ }^{31}$ die Annahme eines gesteigerten Selbstwertgefühls nahelegen bzw. suggeriert mehr oder weniger zutreffend, die collegia seien "Geheimlogen “32 gewesen. Insbesondere das Kultgeschehen, das den einzelnen $\tau \varepsilon \lambda$ oú $\mu \varepsilon v o \varsigma$ begeisterte

28. So etwa in ApUl. met. XI 23, wenn auch ein wenig kokettierend, dargestellt. Der Quellenauszug unterstreicht einen wesentlichen Aspekt der für die Mysterienkulte so typischen Arkandisziplin. Er war für die numinosen Inhalte von herausragender Bedeutung und wird von EDGAR WIND, Heidnische Mysterien in der Renaissance, Suhrkamp Verlag, Frankfurt (Main), S.21. folgendermaßen bewertet: „Doch Geheimhaltung war nicht nur Teil ihrer Definition, sondern trug auch dazu bei, daß sie Ehrfurcht einflößten. Die Tatsache, daß diese erhabenen Offenbarungen nur schwer zugänglich waren, schien ihre Autorität zu erhöhen. Doch wenn diese Autorität spürbar sein sollte, durften die Mysterien nicht völlig verborgen werden: man mußte auch wissen, daß es sie gab."

29. U. a. DiOD. V 49, 5. zeigt, für welche Bereiche in einer samothrakischen Kabiren-Gemeinde die Arkandisziplin galt.

30. Hom. h. Dem. 480-482/486-489. (hrsg. v. Anton Weiner, 1986.) Dass sich die Demetreioi auch im Jenseits als privilegiert verstehen durften, illustriert $u$. a. TrGF IV 847.

31. Dass Kulthandlungen in einem bestimmten Rahmen stattfanden, zu dem man nur als (werdendes) Gemeindemitglied Zugang hatte, geht bspw. aus Liv. XXXIX 8, 5-8. hervor.

32. Neben НDT. VIII 65. unterstreicht besonders ApUL. met. XI 17, dass die sodalitates verschiedentlich ins öffentliche Bewusstsein traten. Dass es sich bei ihnen kaum um subversive Logen o. ä. handelte, die die Öffentlichkeit scheuten, lässt weiterhin folgende Beobachtung von MAnfred Clauss, Cultores Mithrae. Die Anhängerschaft des Mithras-Kultes, Franz Steiner Verlag, Stuttgart, 1992, S.255. erkennen: „Ein gewisser Pallas widmete Mithras eine eigene Darstellung; etwas später wirkte Eubulos, der in mehreren

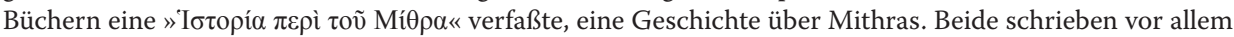
für das stadtrömische Publikum und trugen zweifellos dazu bei, den Kult weiter bekannt zu machen." [Hervorhebung im Original, D. M.]. Ferner spricht insbesondere der Umstand, dass bestimmte Mythologeme der Eleusinischen Mysterien verschiedentlich kommuniziert wurden (z. B. Rezeption ausgesuchter Inhalte

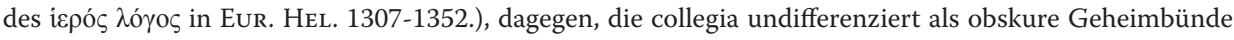
o. ä. zu verstehen. So sagt z. B. KerÉNYI, Eleusis (wie Anm. 19), S.116. über die schließlich von Athen umfassend forcierte Außendarstellung der Eleusinischen Mysterien: „Mit religiöser Mission wäre zuviel gesagt, mit Propaganda etwas Krasses, der heutigen Denkweise vielleicht Entsprechendes, doch in dieser Form kaum Erweisbares. Nur von einer Expansion dürfen wir reden, die in der nichtliterarischen Sphäre festzustellen ist: bezeugt durch die von Athen aus verschickten Vasen, die wohl als Grabgefäße bestellt wurden." 
sowie in ganzheitlicher Weise involvierte, ${ }^{33}$ zeichnete sich durch eine gezielte Spektakularisierung aus. Neben der Außeralltäglichkeit wurde die vermeintliche Exklusivität der collegia buchstäblich dadurch in Szene gesetzt, dass man das bisweilen recht langfristige Initiationsprozedere abgeschlossen hatte $^{34}$ und alsdann vereinzelt der eigene Name wenigstens im kultischen Kontext durch ein (theophores) Hieronym ersetzt wurde ${ }^{35}$ respektive ein Signum hinzutrat. ${ }^{36} \mathrm{Zu}$ ergänzen wäre darüber hinaus etwa, dass sich sodalitates nachts manchmal an relativ unwirtlichen Orten versammelten ${ }^{37}$

33. Recht umfassend stellt BERNHARD BöNISCH, Antike Mysterienkulte - unterwegs auf einer kulturund religionssoziologischen Reise zu den Tiefen menschlicher Existenz, o. V, Passau, 2000, S.57/61. dar, wie die ö $p \gamma 1 \alpha$ die Teilnehmer begeisterten und ganzheitlich involvierten: „Im Mittelpunkt aller Bestrebungen, die mit dem Wunsche der Menschen nach Einweihung zusammenhängen, stand die Hoffnung auf eine persönlichere Beziehung zu und Bindung an den verehrten Gott, ein Faktum, welches die neu entstandenen Mysterienreligionen von der bis dahin tradierten Götterwelt unterschied. [...]. Zentral in den antiken Mysterien, aber auch in unserer heutigen Zeit wieder verstärkt auftretend, ist das sozial-individuelle Verlangen nach umfassenden, ganzheitlichen Sinneseindrücken, und zwar über das gesamte Spektrum menschlich-sensualistischer Wahrnehmungsmöglichkeiten durch Auge (z. B. Kultstatuen), Ohren (etwa Hymnen), Mund (z. B. kultisches Mahl) und Hände (etwa Berührung heiliger Gegenstände) sowie weiterer psychisch-körperlicher Wahrnehmungsmöglichkeiten (etwa Empfinden »heiligen Grauens« im Rahmen des Tests von Mut und Ausdauer des Initianden oder Schmerzempfinden z. B. bei Selbstkastration).“ [Hervorhebung im Original, D. M.]. Bspw. Plut. mor. 352c. gibt Auskunft über den gottesdienstlichen Kern (Dromena, Deiknymena sowie Legomena) einer bestimmten Isis-Gemeinde.

34. An dieser Stelle lässt sich u. a. auf die Eleusinischen Mysterien hinweisen. Diese bestanden schließlich aus der im Anthesterion abgehaltenen $\mu$ únбıৎ. Ihr schlossen sich im Boedromion die $\tau \varepsilon \lambda \varepsilon \tau \alpha i ́$ an. Deren Beginn markierte die feierliche Prorrhesis, von der uns etwa Aristoph. Ran. 354-370. berichtet. Dass eine direkte Verbindung zwischen beiden Vollzügen bestand, geht u. a. aus PLAT. Gorg. 497 c. hervor. Z. B. mit KerÉnyi, Eleusis (wie Anm. 19), S.61. lässt sich der primär auf eine Katharsis abzielende Zusammenhang zwischen den beiden Handlungen wie folgt verstehen: „Agrai und »myesis« tönten nach leiblichen Weihen, Eleusis und »epopteia « nach geistigen."

35. Beispielhaft hierfür Ausführungen von Dorit ENGSTER, Konkurrenz oder Nebeneinander. Mysterienkulte in der hohen römischen Kaiserzeit, tuduv-Verlags-GmbH, München, 2002, S.401f: „In einigen Mysterienkulten war offenbar die Initiation auch mit einer Namensänderung verbunden. Im Dolichenuskult ist besonders das Auftauchen moralisch aufgeladener Namen wie »Vera« oder »Bona« auffällig. Andere Namen wie z. B. »Marinus« oder »Aquila« tauchen ebenfalls häufiger auf. Es ist nicht klar, ob die betreffenden Personen diese Namen auch im täglichen Leben annahmen oder ob sie nur im kultischen Zusammenhang gebraucht wurden. Letzteres ist wahrscheinlicher und zeigt, daß der Myste in dem von ihm gewählten Kult in eine Art Gegenwelt eintrat. Verbunden mit der Initiation in den Kult und der Annahme des neuen Namens war das Versprechen des besonderen Schutzes durch die Gottheit. Im Kybelekult findet sich relativ häufig der Name »Hilarius«, der mit den »Hilaria«, mit der Freude über die Auferstehung des Attis, in Verbindung stehen könnte." [Hervorhebungen im Original, D. M.]

36. Signa (z. B. CIMRM 422.), mit den $\tau \varepsilon \lambda \varepsilon \tau \alpha i ́$ ggf. angenommen, sind Zusatzbezeichnungen. Sie werden i. d. R. dem eigentlichen Namen (direkt) angeschlossen und stehen als solche mit dem Namensträger in Verbindung. So geben sie Auskunft z. B. über dessen Position sowie Funktion(en) im collegium. Überdies dokumentieren sie u. a., welchen Stellenwert die Gemeinde dem collegiatus zuschrieb bzw. wie er selbst speziell von seinen collegiati (prestigeträchtig) wahrgenommen werden wollte. Das von PETER Thrams: Die signa in den Mithrasinschriften. - In: ZPE 9 (1972), S.139-178, hier: S.172. für die Syndexioi Herausgearbeitete kann, entsprechend kontextualisiert, verallgemeinert werden: „Es zeigt sich also, dass die meisten Mystennamen sich entweder auf Mithras selbst oder in irgendeiner Form (Anspielung auf mystische Grade, Tage der Einweihung usw.) auf den Kult des Gottes beziehen. Die übrigen signa drücken z. T. Eigenschaften des Charakters oder sonstige typische Merkmale der Mysten aus."

37. U. a. WERNER PEEK, Griechische Grabgedichte. Griechisch und deutsch, Wissenschaftliche Buchgesellschaft, Darmstadt, 1960, Nr.178. beweist, dass miletische Bacchanten Gottesdienste „im Gebirge“ unweit der Stadt abhielten. 
oder etwa spezielle Lichteffekte Teil der ö $\rho \gamma 1 \alpha$ waren, ${ }^{38}$ zu denen man häufig besondere Gewänder o. ä. trug. ${ }^{39}$

Für das Gemeindeleben ist festzuhalten, dass dieses grosso modo wesentlich vom zielgerichteten Einsatz gerade derjenigen Mitglieder abhing, die Teil der sozialen Führungsschicht in den oppida des Imperium Romanum waren. Genau wie beim weltlichen Dingen verschriebenen Vereinswesen waren also auch im Mysterienwesen neben persönlichen Kompetenzen materielle Ressourcen eminent wichtig. Somit fanden besser situierte sodales hier ebenfalls ein Forum für ihre statusadäquate sowie prestigeträchtige Bewährung respektive für die anzuerkennende wie Anerkennung begründende Abgrenzung von Dritten. Dies fand ganz in Übereinstimmung mit der im griechisch-römischen Altertum geradezu obsessiven Kommunikation der eigenen gesellschaftlichen Position statt. ${ }^{40}$ Hierauf lässt besonders eine Überlegung von MANFred Clauss schließen. Hinsichtlich des Miteinanders der (werdenden) Mithraisten stellt er fest: „Es ist wichtig, hervorzuheben, daß Soldaten, Beschäftigte des staatlichen Verwaltungsdienstes, Sklaven, Freigelassene und einfache römische Bürger als Mysten im Mithras-Kult eine Bestätigung, beziehungsweise Wiederholung eben jener normalen sozialen Erfahrungen fanden, die sie

38. Neben Hippol. ref. V 8, wo über die Eleusinischen Mysterien informiert wird, lässt sich an dieser Stelle etwa mit Johannes Leipoldt, Die Mysterien. - In: Von den Mysterien zur Kirche. Gesammelte Aufsätze, Herbert Reich Evang. Verlag GmbH, Hamburg-Bergstedt, 1962, S.5-50, hier: S.44. zeigen, was Mithraisten tun konnten, um die Herrlichkeit ihres Gottes würdig zu inszenieren: „Lampenfunde deuten darauf hin, daß man teilweise mit einer raffinierten Beleuchtung arbeitet. Öfters gibt es drehbare Kultbilder, mit verschiedenen Darstellungen auf Vorder- und Rückseite; wenn etwa die Vorderseite Mithras auf dem Stiere zeigt, bringt die Rückseite das Bild eines Mahles der Seligen; aber man darf annehmen, daß das sakramentale Mahl der Mithragläubigen sich in ähnlichen Formen bewegt. Vermutlich wird das Kultbild in einem Augenblick der Verdunkelung gedreht, damit die Gläubigen den Eindruck haben, daß ein Wunder erfolgt sei.“

39. Beispielhaft hierfür Eur. Bacch. 821-836.

40. Insbesondere von CIMRM 423. lässt sich ableiten, dass das Engagement gerade besser situierter Mitglieder mitnichten bloß causa sui geschah, und so erklärt etwa BurKerT, Mysterien (wie Anm. 1), S.37: „aber sie stehen mit Rat und Tat für die gemeinsame Sache zur Verfügung, sie setzten ihre Zeit, ihren Einfluß, Beiträge aus ihrem Vermögen dafür ein. Von den Reichen insbesondere erwartet man namhafte Zuwendungen; die Gegengabe besteht in den »Ehren«, die sie dadurch gewinnen: Viele Inschriften dienen diesem Zweck, die »Ehre« der Gönner bekannt zu machen." [Hervorhebungen im Original, D. M.] Hier wird deutlich, dass die Zugehörigkeit zu einem $\theta i ́ \alpha \sigma o \varsigma$ die individuelle Wertschätzung sowohl gemeindeintern als auch, folgt man z. B. ApUl. met. XI 10f, coram publico befördern konnte, indem die persönlichen (im)materiellen Voraussetzungen verschiedentlich prestigeträchtig zur Schau gestellt wurden. Zentrale Prinzipien sozialer Achtung, die so bezeichnend für das Imperium Romanum waren, begegnen uns u. a. in Apul. met. IV 13. Besonders gut fast Werner Dahlheim, An der Wiege Europas. Städtische Freiheit im antiken Rom, Fischer Taschenbuch Verlag GmbH, Frankfurt (Main), 2000, S.113. wesentliche Faktoren zusammen, gleichwohl hier m E. neben Kontextualisierungen Relativierungen angebracht sind: „Das gesellschaftliche und politische Leben gehorchte drei unumstößlichen und erstaunlich konstanten Grundregeln: Die öffentliche Gemeinschaft war unendlich wichtiger als die private Existenz, allein der Besitz qualifizierte für die öffentlichen Ämter, und der wie auch immer erworbene Reichtum schuf durch die noble Art, ihn auszugeben, gesellschaftliche Macht und Anerkennung." Zeigt weiterhin etwa Apul. met. XI 15, dass neben der persönlichen Herkunft sowohl ein standesgemäßes Ethos als auch ein adäquates Bildungsniveau wichtig für das individuelle Ansehen waren, geht bspw. aus Plin. epist. II 6, 1f. und IX 5, 3. hervor, dass generell Rangunterschiede sowohl maßgeblich das soziale Miteinander bestimmten als auch klar kommuniziert wurden. Wie jedoch u. a. Petron. LXXV 10. - LXXVIII 4. illustriert, gab es prinzipiell die Möglichkeit zum gesellschaftlichen Aufstieg. 
tagtäglich außerhalb der Kultstätten machten: [...]“41 Meiner Meinung nach ist dieser Befund, entsprechend kontextualisiert, verallgemeinerbar. Demnach ist das Mysterienwesen nicht nur als Teil des vielgestaltigen kaiserzeitlichen Sinngebungsspektrums anzusehen, sondern es wird deutlich, dass eine direkte Verbindung zwischen der Gesellschaft und dem (individuellen) Glauben sowie dessen Vollzug bestand. Diese Verbindung ist nicht nur auf das Fragen nach der salus rei publicae oder nach dem eigenen Wohlergehen zu beschränken. Vielmehr wird evident, dass normgebende Werte, geheiligt durch die Tradition und den Aufstieg Roms zum unangefochtenen caput mundi geheiligt waren, ${ }^{42}$

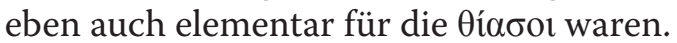

Anhand der (Erst-)Initiationspraxis und des innergemeindlichen Miteinanders möchte ich in meinem Promotionsvorhaben aufzeigen, inwiefern auch im kaiserzeitlichen Mysterienwesen althergebrachte Erwartungen sowie Ansichten zum Tragen kamen. Ich verbinde also bestimmte Aspekte dieser Glaubensoption mit gesellschaftlich anerkannten Konventionen und Vorstellungen in Verbindung und hoffe, so zu einer historisch ausgewogeneren Betrachtung des Mysterienwesens beizutragen. ${ }^{43}$

41. Clauss, Cultores (wie Anm. 38), S.277. Bspw. mit Leipoldt, Mysterien (wie Anm. 37), hier: S.43. lässt sich, Bezug nehmend auf das in ILS 1259. enthaltene „pater patrum“, skizzieren, inwiefern sich im Mithraismus bestimmte Konventionen niederschlugen: „Wenn der Leiter einer Gemeinde Vater heißt (oder gar Vater der Väter, pater patrum), so tritt darin zutage, daß sich alle Teilnehmer als Glieder einer großen Familie fühlen."

42. Dass so mancher Zeitgenosse z. B. einen eklatanten Mangel an religio für die Schrecken, die sich deutlich in den Bürgerkriegen manifestierten, verantwortlich machte, lässt etwa Hor. carm III 6, 1-8. erkennen. So wird allem voran vor dem Hintergrund des von Octavian/Augustus später öffentlichkeitswirksam erklärten „Res publica restituta“ deutlich, weshalb er sich prestigeträchtig sowie System stabilisierend bei der Instandsetzung der Tempel hervortat (z. B. R. Gest. div. Aug. XIX.). Hierauf weist z. B. Heinrich Schlange-Schöningen, Augustus, Wissenschaftliche Buchgesellschaft, Darmstadt, 2012, S.100f. hin: „Augustus hat auf diese religiöse Schuld-Empfindung, die in den Gedichten des Horaz zum Ausdruck kommt, reagiert, indem er seine Neuordnung der »res publica« mit religionspolitischen Maßnahmen begleitete. Angesichts der allgemein propagierten Rückkehr zu alten und besseren Zuständen, mit der die Prinzipatsherrschaft begründet wurde, musste die Religionspolitik des Augustus restaurative Züge tragen, und gleiches gilt auch für seine Gesetzgebung. Allerdings verwandelte sich die römische Religion insofern, als mit der Verehrung des Augustus, wenn sie auch im Osten und Westen des Reiches in unterschiedlicher Weise und in unterschiedlichem Ausmaß praktiziert wurde, ein neuer Aspekt zur herkömmlichen Götterverehrung hinzukam.“ (2012, S.100f.). Besonders deutlich stellen VASSILIS Fyntikoglou u. a., Das römische Gebet. - In: ThesCRA 3 (2005), S.151-179, hier: S.152. die Reziprozität zwischen der salus rei publicae und der korrekten religio dar: „Die römische Religion gestaltete sich nicht aufgrund theoretischer Gedanken, sondern aufgrund angehäufter Erfahrungen, welche die richtigen Kommunikationsmittel zu den Göttern bestimmten [...]; aus diesem Grund ist sie unmittelbar an die sozialen und politischen Entwicklungen gebunden [...]. Das wichtigste Element ist demnach die sorgfältige Ausführung der Opfer, der Gebete, der Gelübde und aller Zeremonien, die zeigen, dass die Menschen die Götter respektieren."

43. Untersuchungen zum gemeindeeigenen Miteinander, das auch in der Öffentlichkeit verschiedentlich kommuniziert wurde (z. B. Apul. met. XI 10f.) und dem Einzelnen die Möglichkeiten sowohl zum Prestigegewinn als auch zur -ostentation bot, halten zu einer kritischen Bewertung von Aussagen an, die die sodalitates, bspw. ausgehend von LSCG 65, Z.15-26, als egalitäre Versammlungen verstehen. So liest man u. a. bei Cumont, orientalischen (wie Anm. 12), S.25: „Unter den Mysten findet man Asiaten und Römer vermischt, Sklaven neben hohen Beamten. Die Annahme desselben Glaubens machte den armen Freigelassenen zum Genossen, bisweilen sogar zum Vorgesetzten des Dekurionen und des »clarissimus«. Alle unterwarfen sich denselben Regeln, alle beteiligten sich an denselben Festen, bei denen die Rangunterschiede einer aristokratischen Gesellschaft wie die Verschiedenheit des Blutes und 
Außerdem dürfte die Auseinandersetzung mit den collegia wichtige Einblicke insbesondere in das politische wie private Leben im Kaiserreich gewähren, das vom Religiösen umfassend durchdrungen war. ${ }^{44}$ Dies betont etwa KAREN PiePENBRINK: „Opfer und Rituale wurden im häuslichen wie im öffentlichen Bereich vollzogen. Auch in der Politik gewannen sie eine wichtige Rolle. Die Römer kannten keine Trennung von Politik und Religion, die dem modernen Verständnis entsprochen hätte. Das Wohlwollen der Götter zu erlangen, war auch für das Gemeinwesen wichtig. ${ }^{45}$

\section{STAND DER FORSCHUNG}

Fragen hinsichtlich des antiken Mysterienwesens sind seit jeher

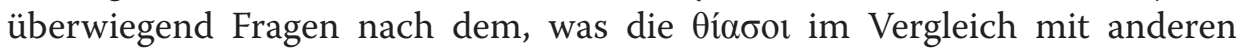
Sinngebungsmodellen auszeichnet. Im Ergebnis präsentiert die Forschung sie weithin als etwas mehr oder minder Fremdes, das exotisch-faszinierend ${ }^{46}$

des Vaterlandes sich verwischten. Es gibt hier keine Rasse und keine Nationalität, keine Behörden und keine Hausherren, keine Patrizier und keine Plebejer, keine Bürger und keine Ausländer mehr, sondern nur noch Menschen, [...]." [Hervorhebung im Original, D. M.]. Allem voran die Prinzipien individueller gesellschaftlicher Wertschätzung, die jahrhundertealten Vorstellungen folgten und auch bestimmend in

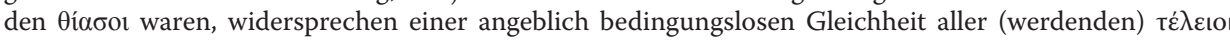
und so erklärt Dieter Zeller, s. v. Mysterien/ Mysterienreligionen. - In: TRE 23 (1994), S.504-526, hier: S.516: „Der Egalitarismus der Mysterien ist manchmal übertrieben worden. Grundsätzlich war zwar jeder Myste durch die Beziehung zu seinem Gott qualifiziert, und die einzelnen Kulte entwickelten ihre eigene Rangordnung. Aber im täglichen Leben blieb alles beim alten [sic!].”

44. Zeigt etwa Cic. nat. deor. II 8, dass die eigene Religiosität wesentlich zur römischen Selbstwahrnehmung gehörte, illustriert z. B. Plut. mor. 1125de. neben deren Allgegenwart sowohl deren Selbstverständlichkeit als auch ihren grundlegenden Stellenwert.

45. Karen Piepenbrink, Konstantin der Große und seine Zeit, Wissenschaftliche Buchgesellschaft, Darmstadt, 2010, S.75. Belegt u. a. CATO, agr., 143, 1f, dass im privaten Raum klar reglementierte Kulthandlungen vollzogen wurden, illustriert dies für den öffentlichen Bereich z. B. LIV., XLI 16, 1-6. Dass es nicht möglich ist, beim zweckgebunden Umgang mit den Göttern problemlos in „privat“ und „öffentlich“ zu unterscheiden, betont bspw. Veit Rosenbeger: Religion in der Antike, Wissenschaftliche Buchgesellschaft, Darmstadt, 2012, S.111f: „Doch zugleich sind die Bereiche des Öffentlichen und des Privaten nicht stets scharf voneinander zu trennen. Nicht alles, was im Haus geschah, war privater Natur; weite Bereiche eines Hauses waren der Öffentlichkeit zugänglich. Dies gilt für die gesamte Antike. Umgekehrt gab es öffentliche Bereiche, die nicht zugänglich waren; im religiösen Feld sind die Tempel zu nennen, die oft genug verschlossen waren. [...]. Zum einen konnte, wer »sacra privata « vollzog, einen Priester um Rat fragen; [...]. Zum anderen waren auch die »sacra publica« für individuelle Initiativen offen. [...]. Viele Rituale hatten eine öffentliche und eine private Seite: Zu Beginn der Saturnalien im Dezember [...] wurde vor dem Saturntempel in aller Öffentlichkeit geopfert, danach spielten sich die Rituale in den einzelnen Häusern ab." [Hervorhebungen im Original, D. M.]

46. Für die Faszination für das Mysterienwesen, die schließlich verstärkt im Kaiserreich besonders stark um sich gegriffen habe, zeichneten z. B. laut TORBEN CHRISTEnsen, Christus oder Jupiter: Der Kampf um die geistige Grundlage des Römischen Reiches, Vandenhoeck \& Ruprecht, Göttingen, 1981, S.33. im Wesentlichen folgende Faktoren verantwortlich: „Der fremdartige und exotische Charakter der orientalischen Religionen erregte keinen Anstoß mehr, sondern im Gegenteil: er faszinierte. Außerdem war man längst zu einer hohen Wertschätzung der »Weisheit der Barbaren« gelangt, denn sie konnte sich auf uralte, heilige Traditionen berufen - und »antiquitas« wurde im Denken der Kaiserzeit als ein fast unfehlbares Wahrheitskriterium betrachtet. Die Mysterienreligionen beschränkten sich nicht nur auf die Riten, die das Heil vermittelten, sondern sie boten auch neue Erkenntnisse des Göttlichen, der Welt und des Menschlichen. An der Spitze dieser Kulte standen oft Priester, die sich schließlich den religiösen Aufgaben hingaben. Sie leiteten die heiligen Kulthandlungen und nahmen sich als Seelenführer des religiösen Lebens des einzelnen an. Hier wurden die Menschen - im Gegensatz zur Staatsreligion - zum Gegenstand persönlicher Fürsorge." [Hervorhebungen im Original, D. M.] 
bzw. zutiefst anrüchig von einem schon im Altertum jahrhundertealten Verstehens-, Vorstellungs- sowie Erwartungshorizont differiere. Das Anliegen, ihr (angebliches) Besonderes näher zu bestimmen, ist ein beständiges Thema in verschiedenen Wissenschaften. Dies zeigt etwa neben einem Aufsatz in einer Fachzeitschrift für Psychiatrie ${ }^{47}$ besonders deutlich der Titel der 1909 erstmals erschienenen Monographie KAREL H. E. DE JONGS: „Das antike Mysterienwesen in religionsgeschichtlicher, ethnologischer und psychologischer Bedeutung".

Die Thesen, die über das Mysterienwesen angestellt werden, beschränken sich nicht nur auf einen phänomenologisch angelegten Vergleich mit den staatstragenden sacra publica bzw. erörtern das Ausmaß einer vermeintlich grundsätzlichen Devianz. Wie im vorangegangenen Abschnitt aufgezeigt wird ihm nämlich großes Interesse bei Reflexionenüber dieEntstehungsbedingungen des Christentums, dessen Entwicklungen sowie Aufstieg zuteil. So steht auch gerade die Suche nach dem Umfang der Ingerenz spezieller Theologumena bzw. Praktiken auf die christlichen Versammlungen schließlich im Zentrum der sog. "Religionsgeschichtlichen Schule“. ${ }^{48}$ Hier wollte man aufzeigen, inwiefern und weshalb diese (angeblich) bewusst Anleihen bei außerchristlichen Quellen gemacht hätten. ${ }^{49}$ Die Theoreme, die in den letzten beiden Jahrhunderten im Zuge interdisziplinärer Studien entworfen wurden, entfalteten eine noch bis in die Gegenwart hineinreichende Wirkmächtigkeit. In Sonderheit lehnte man einhellig die selbst häufig noch bis weit ins 20. Jh. vertretene Frontstellung „Heidentum / Mysterienwesen versus Christentum“ ab. Man war der Überzeugung, das Christentum sei primär in liturgischer

47. Gunter Hofer, Geheimnis und Verheimlichung. - In: Confin. psychiat. 7 (1964), S.234-258.

48. Auf Basis grundlegender Texte behandelt JAAn LAHE, Die „orientalischen Religionen“im Römerreich als ein Problem der Religionsgeschichte. - In: ZfR 20 (2012), S.151-195. eindrucksvoll zentrale Aspekte, die seit jeher mit dem antiken Mysterienwesen verbunden sind. Hierbei setzt er sich in erster Linie umfassend mit den Themen „Religionsgeschichtliche Schule“, „Entwicklung der Mysterienwesen-Forschung seit dem späten 19. Jh. und deren Exponenten“, „'Orient' als Ursprungsregion der Mysterienlehren“ sowie mit der Frage auseinander, inwiefern man von „Mysterienreligionen“ sprechen kann. Neben (vermeintlichen) Charakteristika stellt er so grundlegende Fragen an das Mysterienwesen zusammen, zeichnet dessen Bedeutung im kaiserzeitlichen Synkretismus nach und liefert themenspezifisch weiterführende Literatur. Dass der Beginn breiter angelegter Diskurse über den Einfluss außerchristlicher Inhalte auf das Christentum auch früher datiert werden kann, zeigt etwa CARL CLEMEN, Die religionsgeschichtliche Methode in der Theologie, J. Ricker'sche Verlagsbuchhandlung (Alfred Töpelmann), Gießen, 1904, S.14f: „Wohl aber legt sie [sc. die religionsgeschichtliche Methode] uns die Frage nahe, ob es in seiner "geschichtlichen Ausprägung «, wie überhaupt von seiner Umgebung, so nicht auch von andern Religionen beeinflußt worden sei: [...]. Handelte es sich bei dem letzten Punkt um eine Anschauung, die in dieser Frage erst in neuester Zeit Vertretung gefunden hat, so ist umgekehrt der Versuch, das Christentum zum Teil aus andern Religionen zu erklären, wieder schon im 17. und 18. Jahrhundert gemacht worden. An einzelnen Stellen war das sogar noch früher bereits geschehen - so wurde z. B. die Frage nach dem Platonismus der Kirchenväter schon im 16. Jahrhundert, u. a. auch von »Luther«, ventiliert - aber auf die gesamte Entwicklung des Christentums ist diese Methode doch eben erst vom Deismus angewandt worden." [Hervorhebungen im Original, D. M.]

49. Beispielhaft lassen sich mit CLEMEN (ibid., S.15f.) die Faktoren zusammenfassen, die es rechtfertigen, einen direkten, in seiner Tragweite aber nur schwer einschätzbaren Einfluss außerchristlicher Quellen auf das Christentum zu attestieren: „Mit Bestimmtheit kann nämlich nur dann von fremden Einflüssen auf das Christentum gesprochen werden, wenn folgende - eigentlich selbstverständliche, aber vielfach übersehene - drei Bedingungen erfüllt sind: 1. eine Anschauung darf sich aus den ursprünglichen christlichen Idee schlechterdings nicht erklären lassen; 2. sie muß in einer anderen Religion wirklich nachweisbar sein und 3. es muß sich verständlich machen lassen, wie sie aus dieser in das Christentum überging." 
sowie dogmatischer Hinsicht speziell vom Heidentum her beeinflusst. Schnell war man damit bei der Hand, insbesondere die Mysterienkulte für die rasche Inkulturation des Christentums verantwortlich zu machen. Immerhin könne man z. B. weder das Verständnis vom errettenden Sterben eines einst wiederkehrenden Gottes (sohnes) noch die Taufe respektive Eucharistie als zentrale christliche Initiationssakramente im alttestamentlich-jüdischen Denken verorten. Im Verlauf der adressatenorientierter Christianisierungen seien die ursprünglichen Phänomene weit verbreiteten paganen Ansichten angepasst worden. Diesbezüglich erklärt bspw. HANS vON SODEN: „Was uns die christliche Lehre hoch und schwer macht, hat einmal dazu gedient, sie verständlich und allgemein zugänglich zu machen. Was uns als Mauer und Graben den Weg sperrt, ist einmal Brücke und Tor gewesen. ${ }^{\text {"50 }}$ Dass im Rahmen der Inkulturation des christlichen Kerygmas gezielt Anleihen speziell beim Mysterienwesen gemacht wurden, ließe sich u. a. am scheinbar im Römerbrief (Röm 6,3-11.) enthaltenen Mysterienvokabular erkennen. ${ }^{51}$

Derzeit dominieren zwei Bereiche den wissenschaftlichen Diskurs über das Besondere respektive über das Wesen der Mysterienkulte: Einerseits erfreut sich die Ansicht großer Beliebtheit, es bestünde eine kategoriale Verschiedenheit der sodalitates primär von grundsätzlichen Vorstellungen sowie Kulthandlungen, die dem Bereich der römischen Staatsgötter angehören. Andrerseits bezieht man sich i. d. R. unter dem Eindruck umfassender außenwie innenpolitischer Krisen, die das Kaiserreich schließlich herausforderten, ${ }^{52}$

50. HANS VON SODEN, MYLTHPION und sacramentum in den ersten zwei Jahrhunderten der Kirche. - In: ZNW 12 (1911), S.188-227, hier: S.188. Dass sich die Christen bei der Kommunikation ihrer Glaubensinhalte bewusst einschlägiger Vorstellungen bedienten, die bisweilen direkt mit dem Mysterienwesen in Verbindung standen, geht bspw. aus Clem. Al., protr. XII 119, 1. hervor. Z. B. KLAUCK, Urchristentums (wie Anm. 25), S.128. arbeitet heraus, weshalb die Frage nach der zweckorientierten Adaption außerchristlicher Inhalte im Zuge der Christianisierungen differenziert zu betrachten ist: „Die unbestreitbare Ähnlichkeit mancher Phänomene bleibt unter einer anthropologischen Perspektive in jedem Fall von Interesse: Bestimmte religiöse Verhaltensweisen und Erlebnisformen sind offenkundig im Menschen tief verankert, weil sie mit individuellen und gesellschaftlichen Grunderfahrungen eng zusammenhängen. Es steht zu ihrer Bewältigung nur ein begrenztes Ausdruckspotential zur Verfügung. So groß ist der Vorrat an Gesten und Handlungen nicht, auf die man verfallen könnte. Das gilt insbesondere dann, wenn sich dazu noch ein relativ homogener kultureller Hintergrund ausmachen läßt, und der ist in der antiken Mittelmeerwelt trotz aller ethnischen Vielfalt und politischen Pluralität gegeben. Auch die Mysterienkulte bilden einen untrennbaren Bestandteil des nichtjüdischen Rezeptionshorizonts für die christliche Botschaft." Wie u. a. Clemen, Methode (wie Anm. 48), S.13. zeigt, war auch Vertretern der Religionsgeschichtlichen Schule bewusst, dass das Christentum von Anfang an verschiedentlich mit seiner Umwelt interagierte: „Richtig ist nämlich, [...] daß keine historische Persönlichkeit schlechterdings unabhängig von ihrer Zeit ist, daß vielmehr selbst die originellsten Denker immer gewisse Anschauungen aus ihrer Umgebung aufnehmen."

51. Stellvertretend für neuere Studien, die sich mit der hier thematisierten zweckgebundenen Adaption paganer Vorstellungen durch die Christen beschäftigen, sei CHRIstoph Auffarth, Das Korn der Sterblichkeit: Was Paulus von seinen Korinthern im Demeter- und Kore-Heiligtum gelernt hat. - In: RüPKE, JöRG u. a. (Hrsgg.): Bestattungsrituale und Totenkult in der römischen Kaiserzeit, Franz Steiner Verlag, Stuttgart, 2010, S.113-133. genannt.

52. Als Beispiel für eine umfassende Krise sei hier etwa mit SHA VER., VIII 1-3. auf die sog. „Antoninische Pest" verwiesen. 
auf das persönlichere Miteinander zwischen der Mysteriengottheit und dem (werdenden) Mysten. So habe die Attraktivität der Versammlungen besonders in den begeisternden gottesdienstlichen Handlungen bestanden, die sich Aufmerksamkeit heischend von den staatstragenden und exakt reglementierten

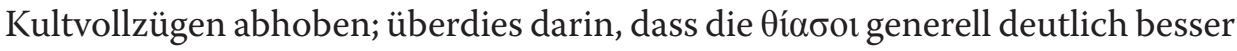
verschiedenen persönlichen Sehnsüchten hätten gerecht werden können. Ihre Anziehungskraft - gerade hieran ließe sich die Alterität ausmachen - habe sowohl in der unverbrüchlichen Zusage diesseitiger Privilegien als auch in konkreten Jenseitsverheißungen bestanden. Anders als die Staatsgötter, die mit keinerlei trostreichen Jenseitsverheißungen aufwarten konnten, ${ }^{53}$ ließen sich die Mysteriengötter nämlich vom individuellen Schicksal jedes einzelnen Gläubigen anrühren. ${ }^{54}$ Darüber hinaus hatten sie ja ihrerseits so manche

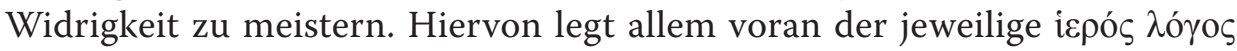
ein beredtes Zeugnis ab. ${ }^{55}$ Die ihnen so zugesprochene "Menschlichkeit"

53. Exempli gratia wird in Sall. Catil. LI 20. neben pessimistischer Skepsis deutlich, dass wohl kaum ein Heide auf ein trostreiches „Danach“ oder göttlichen Beistand beim Sterben hoffte. Nichtsdestotrotz existierte etwa Hom. Od. IV 561-569. zufolge mit dem Elysion ein sagenhafter Ort geradezu paradiesähnlicher Freuden. Zu diesem erlangten aber nur wenige Zugang. Wenn z. B. gemäß ibid. XI 471-491. noch nicht einmal Achilleus, dem antiken Helden schlechthin, (zunächst) ein tröstendes Los im Jenseits beschieden war, wie konnte da der gemeine Sterbliche, geradezu tolldreist, darauf verfallen, auf ein besseres Sein über das Grab hinaus zu hoffen? Dass dies eben kaum der Fall war, mag bspw. Gerhard Pfohl: Griechische Inschriften als Zeugnisse des privaten und öffentlichen Lebens, Heimeran Verlag, München, 1980, Nr.31. verdeutlichen.

54. Dass sich der einzelne mit seinen ganz persönlichen Sorgen, Hoffnungen, Wünschen etc. in den collegia besonders gut aufgehoben verstehen mochte, suggeriert u. a. Apul. met. XI 5f. Mit PiepenbrinK, Konstantin (wie Anm. 45), S.76f. liegt eine Publikation neueren Datums vor, die das hier Zusammengestellte wie folgt erörtert: „Mit der Zeit scheinen die offiziellen Staats- und Stadtgottheiten allein die Bedürfnisse der Menschen im Römischen Reich nicht mehr befriedigt zu haben. Viele wandten sich bereits ab dem 2. Jahrhundert n. Chr. zusätzlich neuen Gottheiten und Kulten zu, die stärker auf ihre persönlichen Belange bezogen waren und damit in höherem Maße individuellen Bedürfnissen entsprachen. [...]. Diese Haltung führte auch dazu, dass ein neuer Typus von Religion großen Zulauf fand, die sog. Mysterienreligionen, die aus dem orientalischen Raum stammten. Sie versprachen dem Einzelnen persönliche Erlösung. [...]. Die Mysterienreligionen unterschieden sich in ihrer Struktur deutlich von der traditionellen römischen Religion. [...]. Die Personen, die sich in die Kulte einweihen ließen, die sog. Mysten, strebten nach persönlicher Nähe zu diesem Gott. Ein solches Nahverhältnis zu einer Gottheit war bislang unbekannt. Die Mysten wünschten von ihr Hilfestellung in ihrer Lebensführung und Erlösung von irdischen Beschwernissen. Auf derartige Erwartungen antwortete die pagane Religion ebenfalls nicht. [...]. Die Methoden, die man verwendete, um sich dem Gott anzunähern, waren verschieden. Meist wurden Praktiken angewendet, die die Mysten in einen ekstatischen Zustand versetzten, in dem sie eine Epiphanie erfuhren, d. h. eine persönliche Erscheinung des Gottes zu erleben meinten. Zum Teil wurde sogar eine Vereinigung mit dem Gott angestrebt."

55. Hier lässt sich als eine Quelle MIN. FeL., XXII 1. nennen. Es begegnet uns, ungeachtet aller Polemik, das Besondere der Mysteriengötter, nämlich Götter in vicissitudine zu sein. Somit spiegelt das im ícpóc $\lambda$ ójo $\varsigma$ tradierte Geschick die menschliche Lebensreise wider. Hierdurch konnte sich der Adorant seiner

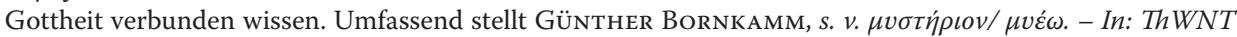
4 (1942), S.809-834, hier: S.811f. die verschiedenen Aspekte des menschlichen Lebens zusammen, das dem jeweiligen Göttermythos zugrunde lag: „Sie sind - zunächst in einem allg. Sinn - leidende Götter; der

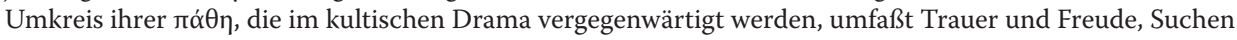
und Finden, Zeugung und Geburt, Sterben und Leben, Anfang und Ende." [Hervorhebung im Original, D. M.]. Mit SCHNeIder, Kulturgeschichte (wie Anm. 6), S.767. lässt sich das womöglich Faszinierendste an den Mysteriengöttern festhalten: „der Gott nimmt das ganze Schicksal des Menschen, also auch Geburt und Tod, auf sich. Ein Gott, der nicht stirbt, weiß ja gar nicht, wie es dem sterbenden Menschen zumute ist." Allerdings ist das "Sterben“ etwa ZELLER, Identifikationsfiguren (wie Anm. 25), S.132. zufolge differenzierter zu betrachten: „manche halten sich auch nur für eine gewisse Zeit in der Unterwelt auf;“ Was das göttliche 
unterschied sie deutlich vom ebenfalls anthropomorph gedachten Jupiter, Mars, der Venus usw. ab. ${ }^{56}$ Der Sterbliche wusste, dass er in ihnen ein verständnisvolles Gegenüber hatte, das sich fürsorglich seiner annahm, ${ }^{57}$ und so kommt z. B. Alexander J. M. Wedderburn zu dem Schluss: „the »humanity" of the mystery-gods, i. e. their having suffered as humans do, at the same time provided the possibility of »identification « and communion and also, paradoxically, made them greater and more majestic than the old gods, so that the relationship between humans and their gods was displayed in the first instance in self-abasement and surrender. ${ }^{458}$ Nichts stand in den óp $ү 1 \alpha$ der persönlichen Gottesbegegnung im Weg und so konnte jeder, sofern er als Adept einst bestimmten Anforderungen genügt hatte, zur Zuversicht schenkenden Epoptie gelangen.

Wie die folgende exemplarische Auflistung einschlägiger Projekte zeigt, lässt sich mein Promotionsvorhaben der aktuellen deutschen Forschungslandschaft zurechnen. Sehr viel versprechend verheißt das interdisziplinäre Erfurter Projekt „Lived Ancient Religion: Questioning 'cults' and 'polis religion"“Projekt zu werden. Neben dem im Internet abrufbaren Profil legen die fünf Teilprojekte (besonders: „Individuelles religiöses Handeln zwischen legitimer Pluralität und Devianz") und die Eröffnungstagung (Mitte Juni 2013), ${ }^{59}$ den Schluss nahe, dass hier weitere Erkenntnisse u. a. über das Mysterienwesen als eine spezielle Form der antiken Suche nach Sinn, Geborgenheit und übernatürlichem Beistand zu erwarten sind. ${ }^{60}$ Diese werden womöglich

"Leiden“ betrifft, das der (werdende) Myste verschiedentlich solidarisch mit dem Gott Heil bringend mitempfand (z. B. Clem. Al. protr. II 12, 2.), ist bspw. mit Burkert, Mysterien (wie Anm. 1), S.64f. eine kritischere Haltung gegenüber entsprechenden Aussagen anzumahnen: „Die Mithrasmysterien heben sich durch die Ausklammerung von Leiden und Klagen im Göttermythos von den anderen Mysterien ab; sie beweisen eben damit, daß Mysterien ohne den Typ des »leidenden Gottes« möglich sind." [Hervorhebung im Original, D. M.]. Um eine grundlegende Vorstellung davon zu bekommen, wie man sich die Solidarität des einzelnen, seine Sympathie mit der Mysteriengottheit vorstellen kann, bieten sich besonders Ausführungen von Wedderburn, Baptism (wie Anm. 27), S.332f. an: „Again, we speak of »identifying ourselves with someone or something in the sense that their interests, their feelings, their experiences or the like become ours, are shared by us. This is the language of »solidarity«. [...]. It is an expression of »sympathy « in the truest sense of the word, a suffering with another, a bearing of some portion of their grief, an acknowledgement that their loss is a shared one. Or we »identify« ourselves with a cause, by making it ours, giving ourselves to its service." [Hervorhebungen im Original, D. M.]. Dass ein wie auch immer zu verstehendes „Leiden“ zentral für das Mysterienwesen war, zeigt etwa SyNEs. Dion, VIII 8.

56. Dass die "Menschlichkeit" der im staatstragenden Pantheon Thronenden weder an die der Mysteriengötter noch an die der griechischen Göttermythen heranreichte, legt u. a. VERSNEL, Umbruch (wie Anm. 15), hier: S.44. nahe: „anders als die griechischen kannten die römischen selten oder nie mythologische oder genealogische Beziehungen: sie wurden weder geboren noch heirateten sie, sie kannten weder Seitensprünge noch Liebeleien, weder Leidenschaften noch Gefühle.“

57. Gerade ApUL. met. XI 25. zeigt, dass der (werdende) Myste sich fortan als immerdar behütet, getröstet, geborgen und wunderbar von seiner Gottheit umfangen wähnte.

58. Wedderburn, Baptism (wie Anm. 27), S.338. [Hervorhebungen im Original, D. M.]

59. Der Tagungsbericht ist abrufbar unter: http://www.uni-erfurt.de/fileadmin/public-docs/MaxWeber-Kolleg/6-pdfs/Tagungen/Tagungsberichte/2013-juli-tagungsbericht-lar.pdf [03/10/13]

60. Verschiedentlich kam während der Konferenz „Sharpening the Knife: Making Religion effective in Everyday Life" das antike Mysterienwesen zur Sprache. Lucinda Dirven etwa setzte sich in ihrem tagungsgruppenspezifischen Eröffnungsvortrag „Imaging Religion in Mithraic Cults: The Case of DuraEuropos" mit Alltagsbezügen auseinander, die sie im Dura-Europos-Kultbild glaubt ausmachen zu können. 
auch vom an der Frankfurter Goethe-Universität implementierten Projekt "Christianisierungen im Römischen Reich" oder durch Arbeiten heraufgeführt, denen man sich in Heidelberg im Rahmen des Exzellensclusters „Asien und Europa im globalen Kontext" widmet. Gleiches mag, so eine entsprechende Ausrichtung stattfindet, für das interdisziplinäre Forschungsvorhaben „Region im Umbruch. Regensburg und das nördliche Raetien von Marc Aurel bis zu Karl dem Großen" der Universitäten Regensburg, Passau und Graz respektive für das Kölner Projekt „Zentrale Einheit und regionale Identität im Imperium Romanum" gelten.

Abschließend lässt sich noch neben einem aktuellen Buchprojekt von JAAN LAHE (,'Die orientalischen Kulte' im Römerreich - Alte Kulte im neuen kulturellen Kontext oder Neuschöpfungen? Die Frage nach der Kontinuität der 'orientalischen Gottheiten' im Römerreich “' ${ }^{61}$ auf diefür November 2013 bis Mai 2014 angesetzte Ausstellung „Imperium der Götter: Isis - Mithras - Christus. Kulte und Religionen im Römischen Reich ${ }^{\text {“62 }}$ hinweisen. Umfassend werden in der Ausstellung im Badischen Landesmuseum Karlsruhe in erster Linie die drei Sinngebungsmodelle thematisiert. Dies soll u. a. fundierte Rückschlüsse auf deren jeweilige Attraktivität ermöglichen. Indem Verbindungen zwischen ausgesuchten Mysterienlehren und dem Christentum aufgezeigt werden, dürfte den sodalitates eine breitere öffentliche Wahrnehmung zuteil werden.

\section{Problemstellung und Methode}

Das meiner Arbeit zugrunde liegende zentrale Problem besteht in erster Linie in der Natur des Verhältnisses zwischen den Mysterienkulten und der kaiserzeitlichen Religion bzw. Gesellschaft. Eine weithin als grundsätzlich angesehene Devianz soll kritisch überprüft werden. Ich möchte also der Frage nachgehen, inwiefern es zulässig ist, die collegia als etwas (kategorial) Fremdes zu betrachten, wovon etwa STEPHANIE von Dobbeler ausgeht: „Mysterien sind Geheimkulte, die, trotz unterschiedlicher inhaltlicher Ausrichtung, einige

Weiterhin referierte sie über die Entstehung der dortigen Mithras-Gemeinde. Durch den regen Austausch mit anderen Tagungsteilnehmern und -teilnehmerinnen erhielt ich neben weiterführenden Hinweisen fruchtbare Kritik und so möchte ich diese Gelegenheit dazu nutzen, mich hierfür stellvertretend bei Marlis Arnhold, Anton Bierl, Lucinda Dirven, Richard L. Gordon, Harry Maier, Eric Rebillard, Jörg Rüpke sowie Katharina Waldner zu bedanken.

61. An dieser Stelle möchte ich Herrn Lahe Dank sagen für die Zusendung seiner Buchskizze $(14 / 07 / 13)$ und den weiterführenden Gedankenaustausch. Hauptziel der Monographie ist es, primär auf Basis ikonographischen, numismatischen sowie archäologischen Materials der Frage nachzugehen, ob die „orientalischen Kulte“ erst im Imperium Romanum entstanden oder schließlich insbesondere durch kulturelle Rahmenbedingungen modifiziert wurden. Lahe konzentriert sich hierbei auf Mithras, Iuppiter Dolichenus, Isis und Magna Mater.

62. Informationen über die Ausstellung, die selbst erklärtermaßen auf Basis eindrucksvoller Leihgaben aus den renommiertesten Museen Europas „erstmals eine umfassende und vergleichende Präsentation der sog. orientalischen Kulte und Religionen“ zeigen will, sind abrufbar unter: http://www.landesmuseum.de/ website/Deutsch/Sonderausstellungen/Vorschau/Imperium_der_Goetter.htm [03/10/13] 
Gemeinsamkeiten auch hinsichtlich des rituellen Vollzugs aufweisen: Sie scheuen die Öffentlichkeit, finden im Verborgenen statt und befinden sich somit im Gegenüber zum öffentlichen Kult im Staat." ${ }^{\text {63 }}$

Weiterhin ist nach der persönlichen Erlebnisqualität des Staatskultes zu fragen. Dieser war bekanntermaßen der salus rei publicae wegen exakt definiert. ${ }^{64}$ Die verschiedenen Vollzüge seien, folgt man z. B. Hendrik S. VERSNEL, "gekennzeichnet durch starke Autonomie der Formel, die ihrerseits einen kühlen, sachlichen, ja nahezu juridischen Charakter trägt." ${ }^{\text {"65 }}$ Dieser ipso iure reglementierte Umgang, so VERSNEL weiter, führte zwangsläufig dazu, dass das Miteinander zwischen den Menschen und den Staatsgöttern

63. Leipoldt, Mysterien (wie Anm. 37), hier: S.14. Zwar verweist LeIPOLDT hier expressis verbis auf die Andersartigkeit dionysischer ö $\rho \gamma(\alpha$, doch ist er von der grundsätzlichen Devianz des Mysterienwesens überzeugt. So schreibt er etwa: „Heute wissen wir, daß jede ungewohnte Frömmigkeitsform und jede neuartige Weltanschauung damit rechnen muß, entstellt und dann mit vergifteten Pfeilen angegriffen zu werden. [...]. Schon damals ist mancher geneigt, alles als wertvoll anzusehen, was weit her ist und eine andere Sprache redet. [...]. Entscheidend ist, daß die Mysterien eine Frömmigkeit begründen, die nicht in den Fesseln eines einzigen Volkstums oder bestimmter Stände steckenbleibt, sondern sich an den Menschen wendet." (ibid., S.16/25/49.)

64. Hier lässt sich auf das genau zu unterscheidende römische Gebet als pflichtbewusster sowie zweckorientierter Teil des staatstragenden ius divinum verweisen. Speziell im öffentlichen Raum war es bspw. Plut., Coriolan, XXV. zufolge exakt reglementiert, konnte man doch man nur mittels des korrekten Ablaufs die pax et venia deum erreichen. Zentrale Aspekte der Formalisierung, durch die sich das in welchem Kontext auch immer gesprochene Gebet auszeichnete und die Sorge u. a. dafür zu tragen hatte, den Kontakt mit den überirdischen Mächten besonders feierlich zu gestalten, fassen etwa FynTIKOGLOU u. a.: Gebet (wie Anm. 42), hier: S.152-154/156/160f. zusammen: „Das traditionelle römische Gebet ist weder abstrakt noch subjektiv und es bringt nicht die persönlichen Anliegen des Betenden zum Ausdruck; es ist vielmehr Teil eines bestimmten Ritus, dessen besonderen Charakter und Beitrag zur »pax deum« es zum Ausdruck bringt. [...]. Denn die Gebete sind jener Teil der Religion, bei dem die Sprache besonders wichtig ist. Die Gebetssprache aber ist formelhaft und somit verbindlich; [...]. Man muss schliesslich [sic!] betonen, dass die Rede der religiösen Zeremonie nicht immer ein Gebet ist, da verschiedene heilige Formeln [...] je nach ritueller Besonderheit gebraucht wurden. Die Breite der religiösen Sprache zeigt das Wort »carmen«, das semantisch mit dem Gebet nicht identisch ist [...]. [...]. Der Römer konnte natürlich überall beten (was vor allem für spontane Gebete von Privatleuten gilt), als wirkungsvoller galten Gebete, welche in Heiligtümern stattfanden. Als heiligster Ort Roms wurde das Kapitol für Gebete bevorzugt [...]. [...]. Die Römer glaubten, dass die Verpflichtungen gegenüber den Göttern erfüllt seien, wenn ein Gebet bzw. jede religiöse Handlung richtig, d. h. nach den bestimmten und erprobten Traditionsregeln, ausgeführt wurde $[\ldots]$. [...]. Die auffallend enge Beziehung zwischen der religiösen und der juristischen Sprache der Römer [...] ist ein Zeichen ihres Konservatismus: Sie übertrugen ihre Anschauung von der Beziehung der Menschen zueinander auf die Beziehung zwischen Menschen und Göttern und benutzten dabei Ausdrucksmittel der archaischen präliterarischen Sprache, die vor allem den praktischen Bedürfnissen der Gemeinschaft entsprach und sich durch ihre Direktheit auszeichnete. [...]. Im römischen Gebet werden alle Beteiligten, Personen wie Sachen, mit absoluter Klarheit beschrieben [...].“ [Hervorhebungen im Original, D. M.]

65. Versnel, Umbruch (wie Anm. 15), hier: S.44f. Dass Exaktheit, die den rechten Ort, den rechten Wortlaut, das rechte Zurüsten, die rechten Gesten etc. mit einschloss, elementarer Bestandteil gerade der römischen Gebetspraxis war, zeigt bspw. CIC., nat. deor., II 10. und wird so auch von FynTIKOGLOU u. a.: Gebet (wie Anm. 41), hier: S.161f. betont: „Die Bedeutung, die die Wirksamkeit der genauen Formulierung für die Römer hatte, wird aus der Tatsache deutlich, dass die Gebete, zumindest bei öffentlichen Kulthandlungen, aus heiligen Büchern [...] vorgelesen wurden, um Fehler beim Rezitieren zu vermeiden: Es genügte nicht, die richtigen Worte zu benutzen, man musste sie auch in der richtigen Reihenfolge und ohne Auslassung aussprechen [...]. Die kleinste Abkehr von dem vorgeschriebenen Text stellte einen Unsicherheitsfaktor für den Erfolg des Rituals dar, das in diesem Fall wiederholt werden musste [...]. [...]. Die Verwendung von Konditionalsätzen [...] und die Verhütungsausdrücke in den »leges sacrae« [...] und »vota« zeigen, dass es sich um ein Element der juristischen Sprache handelt." [Hervorhebungen im Original, D. M.] 
„recht arm an Gefühlsmomenten, Innigkeit und Wärme" "66 war. Gerade das Nachdenken über das, was ein Sterblicher "tatsächlich“ beim Umgang mit den Unsterblichen empfunden haben mag, steht mit einem (vermeintlichen)

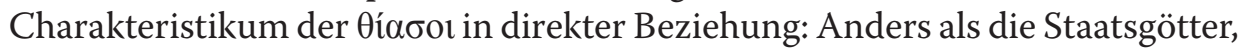
mit denen man äußerst skrupulös interagierte und die ja keinerlei Anteil am Geschick ihrer Gläubigen zu nehmen schienen, ließen sich die Mysteriengötter wie o. a. nicht nur zu einer engen persönlichen Beziehung herab; sie wussten sogar selbst um das, was den Menschen bedrängte.

Kommen wir nun zu den genutzten Quellen. Da es das Ziel meiner Dissertation ist, die Gültigkeit zentraler Aussagen über das Mysterienwesen zu prüfen, die von einer recht umfassenden Andersartigkeit ausgehen, ist weder eine genauere räumliche noch zeitliche Eingrenzung erforderlich. Auch ist wohl die Konzentration auf einen bestimmten Mysterienkult unerheblich. Im Hinblick auf die zugrunde liegenden Quellen spricht nichts gegen die Verwendung exemplarischer Zeugnisse gleich welcher Urheberschaft und Gattung. Allem voran Erkenntnisse über die obligatorische (Erst-) Initiationspraxis bzw. über das Miteinander der (werdenden) collegiati sollen trotz der inhaltlichen Variabilität der Gemeinden und trotz der Tatsache, dass es sich bei ihnen um autonome Bruderschaften handelte, als verallgemeinerbar präsentiert werden.

Neben dem pseudohomerischen Demeterhymnus sei an dieser Stelle auf die von Apuleius von Madaura verfassten „Metamorphoses“ hingewiesen. Dieses Werk, das in den meisten Publikationen über das antike Mysterienwesen herangezogen wird, bietet vielfältige Einblicke in die von mir behandelten Themen. Jan Assmann und Florian Ebeling etwa bezeichnen es als die „wichtigste Quelle für das abendländische Bild der antiken Mysterien im Allgemeinen und der ägyptischen Mysterien im Besonderen [...].“67

Will man Näheres über das gemeindespezifische Kerygma, bestimmte Kultvollzüge usw. erfahren, muss man sich primär christlicher Diffamierungen bedienen, sind doch autobiographische Texte aus Mystenhand relativ selten sind. Darüber hinaus erschwert es die obligatorische Arkandisziplin, detaillierte Einsichten in bestimmte Inhalte zu gewinnen. Jedoch sind die Pamphlete, unbeschadet ihrer Parteilichkeit, Übertreibungen etc., nicht rundheraus abzulehnen, „weil ihre polemische Einstellung “68, so bspw. CARSTEN COlPE über deren Aussagegehalt, „neben der Tendenz zur Entstellung auch den

66. VerSNel, Umbruch (wie Anm. 15), hier: S.44.

67. Jan Assmann u. a., Ägyptische Mysterien. Reisen in die Unterwelt in Aufklärung und Romantik. Eine kommentierte Anthologie, Verlag C. H. Beck oHG, München, 2011, S.29.

68. Carsten Colpe, Die Mithrasmysterien und die Kirchenväter. - In: Boer, Willem den u. a. (Hrsg.): Romanitas et Christianitas. Studia Iano Henrico Waszink. A. D. VI Kal. Nov. A. MCMLXXIII. XIII lustra complenti oblata, North Holland Publishing Company, Amsterdam u. a., 1973, S.29-43, hier: S.29. 
Scharfblick des Gegners erwarten lässt." ${ }^{\text {"99 }}$ Schließlich existieren auch Schriften, für die Heiden verantwortlich zeichnen (z. B. Cicero, Juvenal, Livius etc.) und die sich verschiedentlich mit den sodalitates beschäftigen, überdies vielfältiges archäologisches Material. ${ }^{70}$

Für meine Problemstellung und die aus ihr resultierende typologisierende Methode ist entscheidend - und nach meiner bisherigen Literaturrecherche ist das ein neuer Ansatz -, dass das obligatorische (Erst-)Initiationsprozedere bzw. die Überzeugungen, auf denen es basierte, mit dem mit juridischer Exaktheit geregelten Tieropferkult als (ein) wesentlicher Aspekt der

69. Ibid. Darauf, dass auch beim Umgang mit anderen Textquellen ebenfalls Umsicht geboten ist, macht bspw. Cumont, orientalischen (wie Anm. 12), S.13. aufmerksam: „Aber die Philosophen stellen die fremden Lehren fast niemals objektiv und um ihrer selbst willen dar. Sie verweben sie mit ihren Systemen, denen sie als Beweis oder Illustration dienen sollen, sie verbrämen sie mit ihrer eigenen Exegese oder ertränken sie in transzendenten Kommentaren; mit einem Wort, sie wollen in ihnen alle ihre eigenen Gedanken wiederfinden." Hier bleibt noch u. a. mit Plat. Phaid., 69 b-d. darauf hinzuweisen, dass sich Philosophen, ungeachtet prinzipieller Vorbehalte, bewusst ausgesuchter Inhalte des Mysterienwesens (besonders: individueller Aufstieg zur „tatsächlichen“ Erkenntnis wird durch den figurativen Gebrauch von Bildern und Begrifflichkeiten illustriert, die zentral für die Eleusinischen Mysterien waren) bedienten, um ihre Lehre zu kommunizieren und ihr gleichzeitig einen gewissen Nimbus zu verleihen. Inhalte der Eleusinischen Mysterien, die häufig von Philosophen kritisiert wurden, stellt z. B. WIND, Renaissance (wie Anm. 28), S.13. zusammen: „Doch statt jede Verwandtschaft seiner Philosophie mit solchen Riten von sich $\mathrm{zu}$ weisen, erklärte Platon im Gegenteil, Philosophie selbst sei eine mystische Initiation anderer Art, die durch bewußtes Forschen für wenige Auserwählte das erreiche, was die Mysterien dem gemeinen Volk durch das Schüren von Emotionen vermittelten."

70. Beim Arbeiten mit archäologischen Funden ist äußerste Sorgfalt geboten. Man kann nicht unweigerlich davon ausgehen, dass dort, wo kultspezifische Gegenstände als Zeichen und Gegenstand persönlicher Religiosität vorliegen (z. B. Apul. apol. LV 4/ LVI 1/ 20.), einst „tatsächlich“ geglaubt wurde. Auch spricht kaum etwas wirklich dagegen, dass dort, wo sich nichts Passendes finden lässt, nicht „tatsächlich“ geglaubt wurde. Diesbezüglich möchte ich auf eine Beobachtung von Clauss, Cultores (wie Anm. 32), S.14. verweisen: „Wir müssen uns darüber im Klaren sein, und dies sei trotz der Trivialität einer solchen Bemerkung erwähnt, daß, auf welcher sozialen Ebene auch immer, bessergestellte Gruppierungen in unserem Material eben auch besser repräsentiert sind. Es sind die Erfolgreichen, die wir kennenlernen. Die Stiftung von Altären und Reliefs [...] kostete Geld; dementsprechend war diese Kultpraxis bei den ärmeren Schichten der Anhängerschaft wenig verbreitet." Vorsicht muss man ebenfalls beim Umgang mit christlichen Texten walten lassen. Wie besonders Firm. err. XXII 3. veranschaulicht, konnten sich in Mysteriengemeinden bestimmte Vorstellungen sowie Kultvollzüge ändern. Gerade wenn man auf christliche Quellen zurückgreift, hat man sich hierüber im Klaren zu sein. Besonders ist hierbei danach zu fragen, inwiefern das Behauptete dem in den behandelten collegia Tradierten entspricht und ob es sich nicht vielmehr christlicherseits um eine anachronistische Projektion respektive um eine worin auch immer begründete Neuausrichtung der collegia handelt, gegen die die Christen dann polemisierten. Es steht also die Frage im Raum, inwiefern es zulässig ist, von der Tatsächlichkeit eines konkreten Phänomens auf die genuine Wirklichkeit des nun so Verstandenen zu schließen. Äußerst beachtenswert erscheinen mir an dieser Stelle die Überlegungen Gordons, Mithras (wie Anm. 14), Sp.1000-1002. Die von ihm untersuchten apologetischen Aussagen über Mithras sind m. E. verallgemeinerbar. Allem voran hängt dies damit zusammen, dass es sich bei den Mysterienlehren ja um keine monolithischen Bewegungen handelte, die Überlieferung relativ spärlich ist und die Christen nur bestimmte Phänomene auf Basis ihrer Überlegungen thematisierten: „Sonst wird M. nur von vier christl. Autoren angesprochen, die anscheinend alle ihre Informationen Numenius, Cronius oder Eubulus entnommen u. klar definierte Darstellungsinteressen haben [...]. Ausschließlich bei christlichen Autoren vorkommende Angaben haben deswegen entweder nur lokalen [...] bzw. geringen [...] Erkenntniswert. [...]. Alle drei Autoren [sc. Justin der Märtyrer, Tertullian, Ambrosiaster], besonders Justinus $u$. Tertullian, trachten danach, den M.kult als »Mysterienkult « zu konstruieren, ausgehend von ihrem eigenen Verständnis von regulärer Religion mit sakramentalen Werten u. Praktiken [...]." [Hervorhebung im Original, D. M.] 
sacra publica verbunden werden. $^{71}$ Hierdurch sollte deutlich werden, dass der zweckgebundene Kontakt mit dem Göttlichen jeweils tradierten und gesellschaftlich anerkannten Vorstellungen folgte. Die angeführte Konzentration die $\tau \varepsilon \lambda \varepsilon \tau \alpha$ í bzw. auf die Verallgemeinerbarkeit der Prämissen bietet sich deshalb an, weil sie zentrale Elemente des Mysterienwesens dezidiert hervortreten lassen. Hierzu gehören z. B. die Arkandisziplin, der

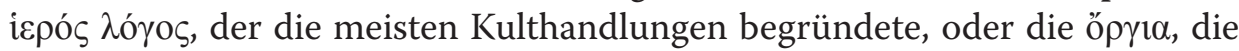
das Individuum verschiedentlich begeisterten sowie unmittelbar einbanden. ${ }^{72}$ Außerdem soll das innergemeindliche Miteinander daraufhin untersucht

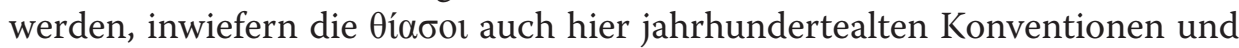
Gegebenheiten folgten.

Neben einer generellen methodischen Vorsicht habe ich auf stete terminologische Exaktheit zu achten. Diese ist sich der Relativität präformierter Beschreibungen bewusst und hält zum umsichtigen Umgang mit entsprechend konnotierten Begrifflichkeiten an, mittels derer etwaige Besonderheiten der sodalitates bestimmt werden sollen. In erster Linie ist diese geforderte differenzierte Betrachtung der Herkunft unseres Wissens aus mehrheitlich christlich bestimmten Quellen geschuldet. Unreflektiert in die Begriffsbildung übernommen, führen sie zu unzulässigen Assoziationen. Gerade dies unterstreicht etwa genau wie die genutzten Quellen die Notwendigkeit interdisziplinären Arbeitens.

\section{GANG DER UNTERSUCHUNG}

Da jeder Glaube und dessen Vollzug weder zeit- noch kontextlos ist, stehen am Anfang meiner Dissertation Reflexionen über Probleme, die sich beim Umgang mit (christlich) präformierten Begrifflichkeiten ergeben. ${ }^{73}$ Dass die (späteren) $\tau \dot{\lambda} \lambda \varepsilon 101$ einem konkreten Hier und Jetzt angehörten,

71. Folgendermaßen stellen etwa MARy BeARD u. a.: Religions of Rome, Bd. 2, Cambridge University Press, Cambridge u. a., 2000, S.148. die Relevanz dieser sorgsam zu begehenden Opfer für den staatstragenden cultus pius deorum sowie überhaupt für die (mittels sacra heraufgeführte) zweckorientierte Kommunikation der Menschen mit den Göttern heraus: „Animal sacrifice, the ritual killing of an animal and the offering to the gods of parts of its body, burnt on the altar, was a (perhaps »the«) central element of Roman ritual." [Hervorhebung im Original, D. M.]

72. Als locus classicus berichtet Plut. fr. 178 Sandbach über die von ambivalenten Erfahrungen geprägte Erlebnisdichte der (Eleusinischen) $\tau \varepsilon \lambda \varepsilon \tau \alpha$ í.

73. Hiermit setzt sich z. B. Christensen, Jupiter (wie Anm. 46), S.24. auseinander: „Es wird im allgemeinen behauptet, der römischen Religion der Kaiserzeit fehle jeder religiöse Wert - sie sei degeneriert und auf eine rein formalistische Einhaltung der althergebrachten Kultriten reduziert. [...]. Diese Darstellung und Beurteilung ist jedoch schief. Man hat nämlich die römische Religion von einem vom Christentum inspirierten Religionsbegriff aus beurteilt, wonach nur das als echte Religiosität zu bewerten ist, was die gesamte Persönlichkeit engagieren und den Intellekt des Menschen, seinen Willen und seine Gefühle auf ein göttliches Wesen mit klar gezeichneten Zügen richten kann. Ein solches Verständnis lag indessen ganz außerhalb des Horizonts römischer Religiosität. Auch in der Kaiserzeit hat sie ihren ursprünglichen 
verlangt entsprechende Klarstellungen. Hernach folgen Aussagen über die Forschungsgeschichte (besonders: Religionsgeschichtliche Schule) und über die aktuelle Forschungslage. Weiterhin ist sicherzustellen, dass man sich über die Bedingtheit der möglichen Eindringtiefe in subjektive (Glaubens-) Erfahrungen im Klaren ist. Ich glaube, dass nur zu einem adäquateren Verständnis eines Phänomens gelangen kann, wer neben der eigentlichen Fremdheit um die Bedingtheit der eigenen Einschätzungen weiß.

Anschließend soll geklärt werden, was in meiner Arbeit unter "Staatsreligion“ verstanden wird. Immerhin geht die Forschung ja weithin von einem grundsätzlichen Gegensatz zwischen dieser und den collegia aus. Zwangsläufig gehört in diesen Abschnitt das phänomenalistische Gottesverständnis. Immerhin wurde ja nur die Gottheit als wahrhaft göttlich angesehen, die ihre Göttlichkeit kundgetan hatte bzw. man glaubte, gerade die Natur vergegenwärtige das Göttliche. Diese Überzeugung begegnet uns auch in sämtlichen Mysterienlehren. Hier wurden die Gottheiten z. B. KARL PRÜMm zufolge, in Sonderheit als „Verkörperungen rein physischer Vitalität $[\ldots]^{{ }^{\top 7} 4}$ präsentiert.

Meine Ausführungen über das phänomenalistische Gottesverständnis bzw. über die Staatsreligion konzentrieren sich auf den blutigen Tieropferkult. Die Heil bringende Antinomie von Werden und Vergehen war Grundlage, die staatstragende pax et venia deum sicherzustellen. Besonderes Interesse wird hier dem juridisch verfassten modus operandi zuteil: Dem Imperium Romanum seien Sicherheit, Stabilität und Wohlstand gewiss, wenn man sich gewissenhaft an das sakrosankte Reglement hielt. Bereits an dieser Stelle möchte ich skizzieren, weshalb es sich anbietet, den klar reglementierten Verkehr zwischen den Sterblichen und Unsterblichen ins Zentrum meiner späteren Ausführungen über die (Erst-)Initiationspraxis zu stellen: Bekanntlich wurde der Adept durch die in der (Erst-)Initiation heraufgeführte Palingenese zum privilegierten véos ảví $\rho$, oder um es mit WALTER BURKERT zu sagen: Das „Zentrum aller Initiation [müssen] Tod und Wiedergeburt sein [...]."75

pragmatischen Charakter bewahrt. Man faßte das Göttliche als eine Kraft auf, deren man sich durch die Einhaltung der vorgeschriebenen Kultriten versichern konnte. Der Vollzug dieses Kultes war die einzige Forderung, die an den Menschen gestellt wurde. Es war deshalb der Wesenszug der römischen Religion, formalistisch zu sein und nichts anderes und nicht mehr als die Wahrnehmung des Kultes zu verlangen damit hatte man seine Schuldigkeit gegenüber den Göttern getan."

74. Prümm: altheidnische (wie Anm. 25), S.305. Beispielhaft charakterisiert Vermaseren, Geschichte (wie Anm. 9), S.54. das geheimnisvolle Zentrum des Mysterienwesens, das Gegenstand u. a. von Min. Fel., XXII 1. ist: „Damit dringen wir zum innersten Kern der antiken Mysterien vor. Diese kreisen letzten Endes alle um das zentrale Problem des Lebens, des Todes und der Auferstehung, so wie man es Jahr für Jahr im ewig wiederholten Zyklus der Natur vor Augen hat."

75. Burkert, Mysterien (wie Anm. 1), S.29. Die von Burkert prononcierte Ambivalenz begegnet uns besonders deutlich in APUL. met. XI 21. und so lässt sich besonders gut mit SAMSON EITREM, Die vier Elemente in der Mysterienweihe. - In: SO 4 (1926), S.39-59, hier: S.39. festhalten: „Fast allen höheren Mysterien ist die Auffassung gemeinsam, daß man sterben muß, um wiedergeboren und so der göttlichen Seeligkeit teilhaftig zu werden. Die Wiedergeburt, die »regeneratio«, um den Ausdruck Tertullians (De bapt. 5) zu gebrauchen, 
Wie deutlich werden soll, kamen Handlungsparadigmata bzw. -überlegungen zum Tragen, die uns m. M. n. auch bei der zweckgebundenen Interaktion mit den Staatsgöttern begegnen. ${ }^{76}$ Somit entsprechen sie einem jahrhundertealten Verstehens-, Vorstellungs- sowie Erwartungshorizont.

Als nächstes setze ich mich mit Definitionen des Mythos auseinander, um für den weiteren Untersuchungsgang eine tragfähige Begrifflichkeit zu erarbeiten. ${ }^{77}$ Immerhin prägte der jeweilige iøpós $\lambda o ́ \gamma o \varsigma$ ja viele der gottesdienstlichen Handlungen und war i. d. R. für die Weihen elementar.

Anschließend widme ich mich dem Werden sowie Sein des altertümlichen Mysterienwesens. Zunächst beschäftige ich mich mit ausgesuchten externen Voraussetzungen, die schließlich im Kaiserreich seine Ausbreitung beförderten. ${ }^{78}$ In diesem Kontext soll auch die Bedeutung des Christentums für

setzt einfach den Tod voraus." Wie etwa Eus. Pr. Ev., II 3, 28. zeigt, setzten christliche Autoren die Ambivalenz herab bzw. verstanden deren grundlegende Relevanz in den Mysterienlehren nicht.

76. Z. B. LIV., XLI 16, 1-3. kann entnommen werden, dass man der Überzeugung war, es herrsche eine direkte Wechselbeziehung zwischen Vergehen und Fortbestehen. Mit der hier thematisierten Ambivalenz setzt sich u. a. Wolfgang Speyer, Töten als Ritus des Lebens. Zum Sinn des Opfers. - In: ders. (Hrsg.): Frühes Christentum im antiken Strahlungsfeld, Kleine Schriften von Wolfgang Speyer, Bd. 2, J. C. B. Mohr (Paul Siebeck), Tübingen, 1999, S.15-49, hier: S.41. auseinander und so deutet er etwa Verg. georg. IV 548558. folgendermaßen: „Wie die Bienen aus den toten Körpern der Rinder entstehen und ihren neuen Kosmos bauen, den Bienenstaat, so hat der Princeps Augustus sein Friedensreich auf den Toten der Bürgerkriege am Ende der Republik errichtet. Der blutige Untergang der Republik ist der Anfang des neuen, augusteischen Friedensreiches." Die Ansicht, es bestehe prinzipiell eine Antithese von Vergehen/ Tod und Werden/ Leben, begegnet uns ebenfalls u. a. in Epikt. diatr. III 20, 4-7. sowie in Joh 12, 24f. Ergo lässt sich wohl von einem Topos sprechen.

77. Es bietet sich hier an, auf Ausführungen von JÖRg RüPKE, Die Religion der Römer. Eine Einführung, C. H. Beck oHG, München, 2001, S.129f. hinzuweisen, um ein grundlegendes Verständnis vom verschiedentlich kommunizierten „(Götter-)Mythos“ zu bekommen: „Nach den archäologischen Quellen waren Rom und Italien wie der gesamte Mittelmeerraum mythengesättigt. Solche archäologischen Zeugnisse sind etwa Kultbilder oder Statuetten, Giebelgruppen an Tempeln, ob gemalt oder als Tonstatuen, kleine Antefixe, also Stirnziegel von Giebeldächern mit kleinen mythologischen Szenen oder Darstellungen von Göttern mit aus der Mythologie bekannten Attributen, schließlich Vasenbilder; später erst folgte die Literatur. [...]. Als Mythen sind nun Erzählungen zu verstehen, die oft in großer zeitlicher Distanz, in einer Urzeit oder sehr fernen Zeit angesiedelt sind, und in ihren Personen-, Sach- und Ereigniskonstellationen das Wertesystem einer Gesellschaft begründen, bestimmte Institutionen und Werte legitimieren und gleichzeitig ein Weltbild vermitteln. [...]. Die hier vorgetragenen Ereignis- und Handlungsfolgen und die darin verwendeten Bilder können auch motivierende Kraft haben." Für das antike Mysterienwesen zeigt etwa Hom. h. Dem. 445-448, dass (Götter)Mythen auf menschliche Erfahrungen und auf menschliches (Nicht-)Verstehen gerade von Naturprozessen rekurrierten. Dass sich (Götter)Mythen auf Reales bezogen, geht u. a. aus MiN. Fel., XXIII 13. hervor. In Verbindung mit Hom. h. Dem. 473-476. illustriert Eun. vit. soph. VII 3, 1f, dass bestimmte Institutionen mythisch legitimiert wurden und bspw. HerodiAN., III 13, 3. unterstreicht, dass (Götter)Mythen auch einen erzieherischen Effekt haben konnten. Wie die Kritik von WALTER BURKERT, Homo necans: Interpretation altgriechischer Opferriten und Mythen, Walter de Gruyter \& Co., Berlin u. a., 1997 an Ном. H. Dem. 445-447. verdeutlicht, stimmt das im (Götter-)Mythos Tradierte nicht immer mit den Realitäten überein (vgl. S.287-289).

78. Z. B. mit Elmar Schwertheim, Mithras. Seine Denkmäler und sein Kult. Raggi Verlag, Feldmeilen, 1979, S.26f. ließe sich hier auf Angehörige des römischen Militärs als Träger bestimmter Sinngebungsmodelle (z. B. CIMRM 1718) und auf die bisweilen dezidierte staatliche Parteinahme (z. B. CIMRM 1698) hierfür (konkret: Mithraismus) verweisen: „Die einflußreichste, durch eine Fülle von Zeugnissen dokumentierte Rolle bei der Verbreitung des Mithraskultes im Imperium Romanum spielten zweifellos die Soldaten bzw. Truppenverbände. [...]. Selbstverständlich war ein tieferes Eindringen des Mithraskultes in das öffentliche und private Leben der Menschen gar nicht möglich, ohne daß Rom und die Kaiser ihre Zustimmung dazu gaben." 
den späteren Untergang - um nicht zu sagen: Vernichtung ${ }^{79}$ - der sodalitates skizziert werden. ${ }^{80}$

Im weiteren Verlauf trage ich Überlegungen darüber zusammen, was die collegia konkret zu bieten hatten. Nun konzentriere ich mich also auf die intrinsische Motivation der (späteren) Mysten. ${ }^{81}$ Meine Ausführungen setzen am persönlichen Moment sowie an den gottesdienstlichen Handlungen an,

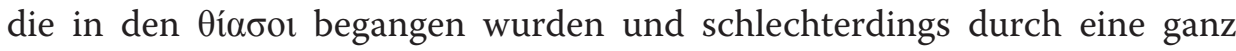
eigene Erlebnisqualität bestachen. Außerdem will ich erörtern, inwiefern in bestimmten gesellschaftlichen Schichten eine gesteigerte Affinität zum Mysterienwesen vorgelegen haben mag. Somit erlaubt gerade dieser Abschnitt Rückschlüsse auf die Frage, inwiefern das innergemeindliche Miteinander gesellschaftliche Konventionen widerspiegelte. Allerdings hat man sich darüber im Klaren zu sein, dass man lediglich Tendenzen grundsätzlicher Natur aufzeigen kann. Dies liegt hauptsächlich sowohl an der persönlichen Dimension des Glaubens als auch daran, dass, so bspw. Hans KLOFT zufolge, weder „»das « allgemeine Profil der Gottheit, »die« typische Kultstätte, »der« typische Gläubige [...] [noch] »die« allgemeine Jenseitshoffnung ${ }^{\text {“82 }}$ existierte.

79. Dass es schließlich zu umfassenden Gewaltexzessen gegen einzelne Versammlungsstätten (konkret: Mithräen) kommen konnte, belegt Hier. epist., CVII 2. Indessen zeigt Socr. H. E., VII 15 sehr deutlich, dass vereinzelt Christen weder vor Mord noch sogar vor Leichenschändung zurückschreckten. Zum umsichtigen Umgang mit den ohnehin nur spärlichen Berichten über Verwüstungen von Versammlungsstätten hält Gordon, Mithras (wie Anm. 14), Sp.1005f. an: „Als Beispiele werden immer wieder dieselben zwei antiken Texte angeführt, als ob sie »typische« Situationen beschrieben; es wird jedoch verschwiegen, dass sie die einzigen ihrer Art sind. [...]. Religiöse Gewalt war jedoch immer lokal begrenzt u. trat nur sporadisch auf [...]. [...]. Eine neuere Studie der Situation in Rom selbst [...] kommt zu dem Schluss, dass von offener christlicher Feindseligkeit keine Rede sein kann, sondern bis zum Dekret des Theodosius iJ. 392 eine ruhige Koexistenz die Regel war [...]. [Hervorhebung im Original, D. M.]. Abschließend bleibt etwa Bezug nehmend auf Cod. Theod. XVI 10, 5. zu betonen, dass im späteren christlichen Kaiserreich mehr oder minder subtil von Staats wegen gegen bestimmte nicht-christliche Praktiken vorgegangen wurde. Hieraus resultierte jedoch zwangsläufig eine starke Beeinträchtigung speziell des Mysterienwesens.

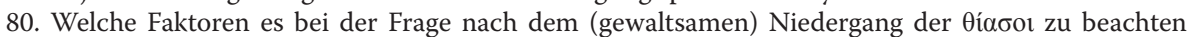
gilt, skizziert u. a. Hans Kloft, Mysterienkulte der Antike. Götter - Menschen - Rituale, C. H. Beck'sche Verlagsbuchhandlung (Oscar Beck), München, 2010, S.109: „Der Niedergang der Mysterienkulte läßt sich also als ein Resultat innerer und äußerer Prozesse verstehen, eben auch von gewaltsamen Umständen und staatlichen Verboten. Es ist nicht einfach, beide Faktoren in ein rechtes Verhältnis zu setzen. Auch verläuft die Entwicklung regional gesehen nicht einheitlich."

81. Neben der Hoffnung auf dies- wie jenseitigen göttlichen Beistand lässt sich noch bspw. CLAuss, Cultores (wie Anm. 32), S.275. entnehmen, dass es manchmal auch eine familieninterne Erwartungshaltung gab, die, etwa vor dem Hintergrund von CIC. leg. II 27, die Mitgliedschaft in einer bestimmten (Mithras-) Gemeinde verlangte: „Bestanden an einem Ort einmal Mithras-Heiligtümer, verbreitete sich der Kult, wie dies nicht anders zu erwarten ist, innerhalb der Familien der Kult-Anhänger; gelegentlich stifteten bis zu vier Familienmitglieder gemeinsam eine Weihegabe. Die Inschriften nennen Brüder, Großväter und Enkel, vor allem aber Väter und Söhne gemeinsam; offensichtlich wurde die Kult-Zugehörigkeit von Generation zu Generation weitergegeben."

82. KLOFT: Mysterienkulte (wie Anm. 80), S.102. [Hervorhebungen im Original, D.M.]. Das von Manfred Clauss, Mithras. Kult und Mysterium, Wissenschaftliche Buchgesellschaft, Darmstadt, 2012, S.25f. für mithräische collegia Herausgearbeitete lässt die generell nötigen Relativierungen sehr deutlich hervortreten: „Die Verbreitung des Kultes reicht von Britannien bis zum Schwarzmeergebiet, vom Rhein bis an den Nil, und dies über beinahe drei Jahrhunderte hinweg, in denen sich die Bedingungen des »Imperium Romanum« veränderten. Das bedeutet, daß der Kult, wenn auch die Hauptlehren und die wichtigsten 
Schließlich soll ebenfalls anhand der obligatorischen (Erst-)Initiationspraxis herausgearbeitet werden, inwieweit ausgewählte tradierte Vorstellungen elementare Bestandteile des kaiserzeitlichen Mysterienwesens waren. Neben dem $\mathrm{m}$. E. allgemeingültigen modus operandi und den Vorstellungen, auf denen er basierte, möchte ich unter dem soeben skizzierten Vorbehalt die mit sämtlichen $\tau \varepsilon \lambda \varepsilon \tau \alpha i ́$ wohl verbundenen grundsätzlichen Hoffnungen der Adepten zusammentragen. Somit wird neben der Heil bringenden Dimension der (rangspezifischen) Weihen dargelegt, inwiefern sie jeweils eine "grundlegende Änderung des religiösen und gesellschaftlichen Status [...]“83 nach sich zogen. Schließlich möchte ich aufzeigen, weshalb der Ausdruck

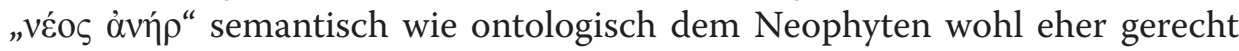
wird $^{84}$ (anders etwa Wilhelm Bousset ${ }^{85}$ Martin Dibelius, ${ }^{86}$ Richard REITZENSTEIN ${ }^{87}$ ).

Im Schlusskapitel möchte ich das Erarbeitete zusammenfassen und zeigen, woraus die z. T. heute noch vorherrschende Gleichung „Mysterienkulte =

Elemente überall gleich waren, zahlreiche Wandlungen mitgemacht hat. [...]. Über den Mithras-Kult als einheitliche Religion zu sprechen, ist daher schwierig; es vereinfacht die Sachlage, aber es verfälscht sie. [...]. Es gab eine Fülle von lokalen und regionalen Besonderheiten und Einflüssen, die am Rhein anders aussahen als am Nil. [...]. Wenn eine Religion sich auf Reisen macht, sich in den unterschiedlichsten Regionen und sozialen Schichten ausbreitet, dann bleiben oft nur noch die Worte und Bilder gemeinsam, nicht aber die Inhalte und die Substanz; denn die Bedeutung eines Symbols ergibt sich aus seiner Umgebung. Eine vorwiegend soldatische Gemeinschaft artikulierte andere Interessen, betonte andere Akzente des Kultes, deutete Zeichen anders als etwa eine Gemeinschaft, in der Sklaven überwogen. Zweifellos waren nur die wenigsten Mithras-Anhänger in der Lage, die Schriften Platons zu lesen. Es macht ferner einen Unterschied, ob ein solcher Kult innerhalb einer urbanen Gesellschaft in Rom oder Ostia oder von Bauern in den Bergen im Inneren des heutigen Jugoslawien gepflegt worden ist. Schließlich schaffen auch die Einflüsse anderer Kulte oder religiöser Strömungen Unterschiede, je nachdem, wie intensiv diese rezipiert werden." [Hervorhebung im Original, D. M.]

83. Eliade, Wiedergeburt (wie Anm. 22), S.11.

84. Dass nicht eindeutig bestimmbar ist, wie man den Status des $\tau \varepsilon \lambda$ ov́ $\mu \varepsilon v o \varsigma$ am besten beschreiben kann, zeigt u. a. Rudolf Bultmann, Das Urchristentum im Rahmen der antiken Religionen, Deutscher Taschenbuch Verlag \& Co. KG, München u. a., 1992, S.198: „Diese Rückkehr [sc. Palingenese des Isiasten Lucius] ist auch als Wiedergeburt bezeichnet worden. Der »Wiedergeborene« - beziehungsweise - der »Verwandelte«, der »Vergottete«, der »Verklärte« - trägt nun gleichsam die »Arznei der Unsterblichkeit« in sich. " [Hervorhebungen im Original, D. M.]

85. Diesbezüglich erklärt Wilhelm Bousset, Christentum und Mysterienreligion. - In: ThR 15 (1912), S.41-61, hier: S.45. etwa: „; er [sc. der Isiakos] hat die Götter von Angesicht zu Angesicht geschaut, und dieses Schauen der Gottheit wird (wie in sämtlichen Mysterien) den Sinn haben, daß durch es der Myste zum Gott selbst erhoben wird. Die »Epoptie«, die heilige Schau, bedeutet nämlich die Verwandlung in die Gottheit." [Hervorhebung im Original, D. M.]

86. Diesbezüglich erklärt Martin Dibelius, Die Isisweihe bei Apuleius und verwandte InitiationsRiten. - In: Bornkamm, Günther (Hrsg.): Botschaft und Geschichte. Gesammelte Aufsätze von Martin Dibelius, Bd. 2, J. C. Mohr (Paul Siebeck), Tübingen, 1956, S.30-79, hier: S.49. etwa: „So wird er [sc. Lucius bei seiner Aufnahme unter die Isiakoi von Kenchreä], durch alle kosmischen Sphären dringend, der kosmischen Allherrscherin Isis ähnlich, so wird er vergottet."

87. Diesbezüglich erklärt Reitzenstein, Mysterienreligionen (wie Anm. 18), S.26. etwa: „Das [sc. besonders die dem Mysten gewisse Emanzipation vom Schicksal] wird begreiflich, wenn wir im Tageskult des ägyptischen Priesters sehen, wie jede Kulthandlung eigentlich ein Zauber ist, und bedenken, daß die unlösliche Verbindung von übernatürlichem Wissen und Wunderkraft, die jetzt im Hellenismus als selbstverständlich gilt, ihre einfachste Erklärung in jener Grundanschauung der Mysterien findet, daß sie mit Gott vereinen und zu Gott machen." 
Geheimlogen ${ }^{\text {"88 }}$ resultiert. Überdies sollen weiterführende Fragen an den Untersuchungsgegenstand gestellt werden. Abschließend möchte ich auf ein Aperçu von Thassilo von Scheffer hinweisen. M. E. trägt es besonders deutlich den generellen Bedingtheiten Rechnung, die so bezeichnend für den Umgang mit dem antiken Mysterienwesen sind: „Wir müssen uns also bescheiden, daß ein Bericht von Mysterien immer nur im Kreis um einen unbekannten Mittelpunkt geht, und daß wir diesen, selbst wenn man ihn uns nennen würde, gar nicht verstehen könnten, eben weil er nicht Verstandessache ist. ${ }^{\text {“89 }}$

\section{Literatur in Auswahl}

Alföldy, GÉZA: Die Krise des Imperium Romanum und die Religion Roms. - In: Eck, Werner (Hrsg.): Religion und Gesellschaft in der römischen Kaiserzeit, Kolloquium zu Ehren von Friedrich Vittinghoff, Böhlau Verlag GmbH \& Cie., Köln u. a., 1989, S.53-102.

Assmann, Aleida u. a.: Mythos. - In: HrwG 4 (1998), S.179-200.

Auffarth, Christoph: „Religio Migrans”: Die „orientalischen Religionen” im Kontext antiker Religion. Ein theoretisches Modell. - In: Bonnet, Corinne u. a. (Hrsgg.): Religioni in contatto nel mediterraneo antico. Modalità di diffusione e processi di interferenza. Atti del $3^{\circ}$ Colloquio su „Le Religioni orientali nel mondo greco e romano", Loveni di Menaggio (Como), 26 - 28 Maggio 2006, Accademia editoriale, Pisa u .a., 2008, S.333-363.

Berking, Helmut H: Schenken. Zur Anthropologie des Gebens, Campus Verlag GmbH, Franfurt (Main) u. a., 1996.

Bowden, Hugh: Mystery Cults of the Ancient World, Thames \& Hudson Ltd., London, 2010.

Burkert, WALTER: Initiation. - In: ThesCRA 2 (2004), S.91-124.

CANCIK, Hubert: „Occulte adhuc colunt". Repression und Metamorphose der römischen Religion in der Spätantike. - In: Kippenberg, Hans G. u. a. (Hrsgg.): Secrecy and Concealment, Studies in the History of Mediterranean and Near Eastern Religions, E. J. Brill, Leiden, 1995, S.191-201.

88. Dies legen u. U. Überlegungen von Johannes KLOPF, Magical-mystery-Tour zum „unsagbaren Mädchen" in Eleusis. - In: Sonntagbauer, Wolfgang u. a. (Hrsgg.): Welt - Seele - Gottheit. Festschrift für Wolfgang Speyer zum 80. Geburtstag, F. Berger u. Söhne Gesellschaft m. b. H, Salzburg u. a., 2013, S.94-112, hier: S.101/106. nahe: „Ein besonderes Kennzeichen der eleusinischen Mysterien ist, dass über den Ritus absolute Verschwiegenheit herrschte, ein Verrat an den Mysterien wurde mit dem Tode bestraft; daher sind die Riten praktisch unbekannt. [...]. Das Mysterium von Eleusis war das bestgehütete Geheimnis der Antike." Umso drängender mögen entsprechende Eindrücke gerade dann werden, versteht man die antiken Mysterienlehren bspw. mit PAUl VeYne: Die griechisch-römische Religion. Kult, Frömmigkeit und Moral, Philipp Reclam jun. GmbH E Co., Stuttgart, 2008, S.112. als „Freimaurerei des Übernatürlichen.“

89. Thassilo von Scheffer, Hellenistische Mysterien und Orakel, W. Spemann Verlag, Stuttgart, 1940 , S.11. 
Colpe, CARSten: Was macht eine Erfahrung zu einer religiösen Erfahrung? Überlegungen aus der Sicht der Religionswissenschaft. - In: Ricken, Friedo (Hrsg.): Religiöse Erfahrung. Ein interdisziplinärer Klärungsversuch, W. Kohlhammer GmbH, Stuttgart, 2004, S.56-68.

Gennep, Arnold van: Übergangsriten, Campus Verlag, Frankfurt (Main), 2005.

Gladigow, Burkhard: Naturae deus humanae mortalis. Zur sozialen Konstruktion des Todes in römischer Zeit. - In: Stephenson, Gunther (Hrsg.): Leben und Tod in den Religionen., Symbol und Wirklichkeit, Wissenschaftliche Buchgesellschaft, Darmstadt, 1994, S.119-133.

Griffiths, John G.: Apuleius of Madauros: The Isis-Book (Metamorphoses, Book XI). Edited with an Introduction, Translation and Commentary by J. Gwyn Griffiths, E. J. Brill, Leiden, 1975.

Herz, Peter: Gab es eine religiöse Grundüberzeugung?. - In: Barceló, Pedro A. (Hrsg.): Religiöser Fundamentalismus in der römischen Kaiserzeit, Franz Steiner Verlag, Stuttgart, 2010, S.57-80.

KirChgäSSner, Alfons: Die mächtigen Zeichen. Ursprünge, Formen und Gesetze des Kultes, Herder, 1959, Basel u. a.

Kloppenborg, John S.: Collegia and „Thiasoi”. Issues in Function, Taxonomy and Membership. - In: ders. u. a. (Hrsgg.): Voluntary Associations in the Graeco-Roman World, Routledge, London, 1996, S.16-30.

LüDEMANN, GERD: Die "Religionsgeschichtliche Schule" und die Neutestamentliche Wissenschaft. - In: ders. (Hrsg.): Die "Religionsgeschichtliche Schule. Facetten eines theologischen Umbruchs, Peter Lang GmbH. Europäischer Verlag der Wissenschaften, Frankfurt (Main), 1996, S.9-22.

Mauss, MarCEL:Die Gabe. Form und Funktion des Austauschs in archaischen Gesellschaften, Suhrkamp, Frankfurt (Main), 1968.

Nedelmann, Brigitta: Geheimnis - Ein interaktionistisches Paradigma. In: Vorgänge 78 (1985), S.38-48.

Rives, James B.: Religion in the Roman Empire, Blackwell Publishing, Malden (MA) u. a., 2007.

Rohde, Dorothea:Zwischen Individuum und Stadtgemeinde. Die Integration von "collegia“ in Hafenstädten, Verlag Antike e. K., Mainz, 2012.

Roloff, DieTrich: Gottähnlichkeit, Vergöttlichung und Erhöhung zu seligem Leben. Untersuchungen zur Herkunft der platonischen Angleichung an Gott, Walter de Gruyter \& Co., Berlin (West), 1970.

RÜPKE, JÖRG: Von Jupiter zu Christus. Religionsgeschichte in römischer Zeit. Wissenschaftliche Buchgesellschaft, Darmstadt, 2011.

Sfameni, Carla: Isis, Cybele and other oriental Gods in Rome in Late Antiquity. "Private” Contexts and the Role of senatorial Aristocracy. - In: Mastrocinque, Attilio u. a. (Hrsgg.): Demeter, Isis, Vesta, and Cybele. Studies in Greek and Roman Religion in Honour of Giulia Sfameni Gasparro, Franz Steiner Verlag, Stuttgart, 2012, S.119-138. 
Simms, Robert M.: Myesis, Telete, and Mysteria. - In: GRBS 31 (1990), S.183195.

Snoek, Johannes A. M.: Initiations. A Methodological Approach to the Application of Classification and Definition Theory in the Study of Rituals, Dutch Efficiency Bureau, Pijnacker, 1987.

STARK, IsOlde: Religiöse Konflikte in Rom durch neue Götter und Kulte (von der Entstehung des Imperium Romanum bis in die Spätantike). - In: Rüpke, Jörg (Hrsg.): Antike Religionsgeschichte in räumlicher Perspektive. Abschlussbericht zum Schwerpunktprogramm 1080 der Deutschen Forschungsgemeinschaft "Römische Reichsreligion und Provinzialreligion", Mohr Siebeck, Tübingen, 2007, S.183-194.

Stausberg, Michael: Religion: Begriff, Definitionen, Theorien. - In: ders. (Hrsg.): Religionswissenschaft, Walter de Gruyter GmbH \& Co. KG, Berlin u. a., 2012, S.33-47.

TAKÁCs, SARolta A.: Isis and Sarapis in the Roman World, E. J. Brill, Leiden u. a., 1995.

Widengren, Geo: Some Reflections on the Rites of Initiation (in the Light of the Papers presented at Strasburg). - In: Bleeker, Claas J. (Hrsg.): Initiation. Contributions to the Theme of the Study-Conference of the International Association for the History of Religions held at Strasburg, September 17th to 22nd 1964, E. J. Brill, Leiden, 1965, S.287-309.

Wiens, Devon H.: Mystery Concepts in Primitive Christianity and its Environment. - In: ANRW II 23/ 2 (1980), S.1248-1284.

Willoughby, Harold R.: Pagan Regeneration. A Study of Mystery Initiations in the Graeco-Roman World, The University of Chicago Press, Chicago (IL), 1974. 